\title{
TOPOLOGICALLY DEFINED CLASSES OF GOING-DOWN DOMAINS
}

BY

IRA J. PAPICK(1)

\begin{abstract}
Let $R$ be an integral domain. Our purpose is to study GD (going-down) domains which arise topologically; that is, we investigate how certain going-down assumptions on $R$ and its overrings relate to the topological space $\operatorname{Spec}(R)$. Many classes of GD domains are introduced topologically, and a systematic study of their behavior under homomorphic images, localization and globalization, integral change of rings, and the " $D+M$ construction" is undertaken. Also studied, is the algebraic and topological relationships between these newly defined classes of GD domains.
\end{abstract}

1. Introduction. The main purpose of this paper is to build upon the studies of Dobbs [5] on going-down extensions and going-down domains, by attacking the problems with a different motivating force. Whereas much of [5] was motivated by flatness, the present work has a topological stimulus. In each of the following sections, we introduce and study new topologically defined classes of going-down domains by considering how various going-down conditions on a domain $R$ and its overrings relate to conditions on the topological space $\operatorname{Spec}(R)$. In each section, we present relevant definitions and notation, although we assume to some extent that the reader is familiar with [3], [4], [5], [6] and [7].

2. Going-down domains and $i$-domains. This section introduces and develops notation and results, some of which we believe to be intrinsically interesting, which will be used continually in the following sections. Many of the lemmas and propositions are straightforward and are stated without proof.

Recall from [5] and [6] that a (commutative integral) domain $R$ is called a going-down domain (written $R$ is GD) in case $R \subset T$ satisfies going-down for each domain $T$ containing $R$; and $R$ is said to be treed if $\operatorname{Spec}(R)$, as a partially

Presented to the Society, January 23, 1975 ; received by the editors March 4, 1975. AMS (MOS) subject classifications (1970). Primary 13A15, 13F05; Secondary 54C10. Key words and phrases. Going-down, treed, mated, open, propen, branch, G-domain, fiber, trunk, vertex.

(1) This work is a portion of the author's Ph.D. thesis written under the direction of Professor David E. Dobbs at Rutgers University. The author wishes to thank Professor Dobbs for his continual assistance and patient guidance in the preparation of this manuscript. 
ordered set under inclusion, is a tree. In [5, Theorem 2.2], it is shown that a GD domain must be treed; an example of Lewis, described in [28] shows that the converse need not be true.

As in [25], an extension $R \subset T$ of domains is said to be open if $\operatorname{Spec}(T)$ $\rightarrow \operatorname{Spec}(R)$ is an open map. (Unless otherwise specified, the map $\operatorname{Spec}(T) \rightarrow$ $\operatorname{Spec}(R)$ is the contraction map.) It is well known (cf. [16, Proposition 1.10.13 (a) and $\left.\left(b^{\prime}\right)\right]$ ) that any open extension satisfies GD; throughout this paper, we exploit this sufficient condition continually.

As in [3], we say that $R \subset T$ is mated if each prime ideal $P$ of $R$ such that $P T \neq T$ is unibranched in $T$; a domain $R$ is said to be mated if $R \subset T$ is a mated extension for each domain $T$ containing $R$ and contained in the quotient field of $R$. In [5, Proposition 3.6], Dobbs shows that an integrally closed domain is Prüfer if and only if it is mated. Call an extension $R \subset T$ an injective extension (and write: $i$-extension) if $\operatorname{Spec}(T) \rightarrow \operatorname{Spec}(R)$ is injective, and say that a domain $R$ is an $i$-domain if $R \subset T$ is an $i$-extension for each domain $T$ containing $R$ and contained in the quotient field of $R$. It is clear that any mated extension is an $i$-extension; Example 2.3 shows that the converse is not generally true. Corollary 2.11 will show that the notions of mated domain and $i$-domain coincide.

A related class of domains consists of the so-called inc-domains (defined below). To some extent our work on them has been anticipated by Gilmer ([11, Theorem 16.10 and Theorem 22.2] , [13, seventh page, LL. 11-13]), whose comments are extended and clarified below.

Throughout this paper, $R$ denotes a domain and $R \subset T$ denotes an extension of domains. We say that $T$ is finitely generated over $R$ if it is so as a ring over $R . \bar{R}$ denotes the integral closure of $R$, and $\mathrm{gf}(R)$ is the quotient field of $R$. The notations for inclusion and proper inclusion are $\subset$ and $\subsetneq$, respectively. By an overring (respectively, proper overring) of $R$, we mean any domain $T$ satisfying $R \subset T \subset \mathrm{qf}(R)$ (respectively, $R \subsetneq T \subset \mathrm{qf}(R)$ ). As in [18, p. 28], the properties of going-up, lying over, and incomparability are denoted by GU, LO, and INC, respectively. A domain is herein called local (respectively, semilocal) in case it possesses only one (respectively, finitely many) maximal ideal(s). Finally, $\operatorname{dim}(R)$ denotes the Krull dimension of $R$. Any unexplained terminology is standard, as in [18] or [11].

PROPOSITION 2.1. If $R$ is $G D$ and each overring of $R$ is treed, then $R / P$ is $G D$ for each $P \in \operatorname{Spec}(R)$.

Proof. In order to prove that $R / P$ is GD, $[6$, Theorem 1] shows that it suffices to establish that $R / P \subset T$ satisfies GD for each valuation overring $T$ of $R / P$. Since $\mathrm{qf}(R / P)$ is canonically isomorphic to $R_{P} / P R_{P}$, any element $P+r$ of $R / P$ may be identified with its image $P R_{P}+r / 1$ in $R_{P} / P R_{P}$. By means of this 
identification, we obtain an overring $S$ of $R$ which is contained in $R_{P}$ and satisfies $T=S / P R_{P}$.

Let $P_{1} / P \subset P_{2} / P$ be prime ideals of $R / P$ and $Q_{2} / P R_{P}$ a prime ideal of $T$ lying over $P_{2} / P$. Without loss of generality, $P \neq P_{1}$. Now, $P_{1} \subset P_{2}$ are primes of $R$, and $Q_{2}$ is a prime of $S$ lying over $P_{2}$. Since $R$ is GD, there exists a prime $Q_{1}$ of $S$ such that $Q_{1} \subset Q_{2}$ and $Q_{1}$ lies over $P_{1}$. Let $M / P R_{P}$ be the maximal ideal of $T$ and observe that $Q_{1} \subset M$. As $S$ is treed, $Q_{1}$ compares with $P R_{P}$. We claim that $P R_{P} \subset Q_{1}$. If not, then

$$
P_{1}=Q_{1} \cap R \subset P R_{P} \cap R=P,
$$

a contradiction. Then $Q_{1} / P R_{P} \cap R / P=P_{1} / P$, whence $R / P \subset T$ satisfies GD, as required.

We now begin to investigate the relationships between GD domains, $i$-domains and mated domains.

Let us first note that if $R$ is an $i$-domain, then each overring enjoys the same property, as well as $R / P$ for each $P \in \operatorname{Spec}(R)$. Also, an easy calculation shows that the " $i$-domain" property is a local property.

Lemma 2.2. Assume that $R \subset T$ satisfies $G D$. Then $R \subset T$ is an i-extension if and only if $R \subset T$ is mated.

EXAMPLE 2.3. The following is an example of an $i$-extension that neither is mated nor satisfies GD. Let $R$ be a 2-dimensional Noetherian domain and let $P$ be a height 2 prime of $R$. Then by a result of Chevalley (cf. [19, Theorem 261]), there exists a discrete (rank 1 ) valuation ring $V$ containing $R$ with maximal ideal $M$, such that $M \cap R=P$. Then $R \subset V$ is an $i$-extension but is neither mated nor GD.

REMARK 2.4. It is easy to construct an extension of domains $R \subset T$ satisfying GD and failing to be mated. (Cf. Example 2.17, for such an example in which $T$ is an overring of $R$.) By Lemma 2.2, no such extension $R \subset T$ can be an $i$-extension; nor can such $T$ be a flat overring of $R$.

Indeed, if $T$ is a flat overring of $R$, then $R \subset T$ is mated. To show this, take $P \in \operatorname{Spec}(R)$ such that $P T \neq T$. Then [30, Theorem 2] implies that $T \subset R_{P}$, thereby producing a prime of $T$ lying over $P$. As for uniqueness, if $Q \in \operatorname{Spec}(T)$ with $Q \cap R=P$, then [30, Theorem 2] gives $T_{Q}=R_{P}$, forcing $Q=P R_{P} \cap T$.

LEMMA 2.5. Let $R \subset T$ be an i-extension, with $T$ finitely generated over $R$ and $R$ integrally closed in $T$. Then $R \subset T$ is open.

Proof. This follows directly from a corollary of Zariski's main theorem [29, Corollaire 2, p. 42].

COROLlary 2.6. Same hypotheses as above. Then $R \subset T$ is mated. 
Proof. Apply Lemma 2.2.

Proposition 2.7. Let $T$ be finitely generated over $R$. If $R \subset S$ is an i-extension for each $S$ satisfying $R \subset S \subset T$, then $R \subset T$ is open.

Proof. Let $R^{\prime}$ be the integral closure of $R$ in $T$. Then by Lemma 2.5, $R^{\prime} \subset T$ is open. Moreover, [2, Remark (2), p. 329] shows that the continuous bijection $\operatorname{Spec}\left(R^{\prime}\right) \rightarrow \operatorname{Spec}(R)$ is a closed map, hence a homeomorphism. Thus, as a composite of open extensions, $R \subset T$ is open.

COROLlaRY 2.8. Let $R$ be an i-domain and $T$ a finitely generated overring of $R$. Then $R \subset T$ is open.

COROLlaRy 2.9. If $R \subset S$ is an i-extension for each $S$ satisfying $R \subset S$ $\subset T$, then $R \subset T$ satisfies $G D$.

Proof. By [25, Lemma 3], we may assume $T$ is finitely generated over $R$, in which case we are done by an appeal to Proposition 2.7 .

Proposition 2.10. If $R \subset R[u]$ is an i-extension for each $u \in T$, then $R \subset T$ is mated.

Proof. We claim that $R \subset S$ is an $i$-extension for each $S$ satisfying $R \subset$ $S \subset T$. If not, there exist distinct primes $P, Q$ of $S$ such that $P \cap R=Q \cap R$. Without loss of generality, choose $u \in P \backslash Q$. Then $P \cap R[u]$ and $Q \cap R[u]$ are distinct primes of $R[u]$, each contracting to $P \cap R$, and hence contradicting the assumption that $R \subset R[u]$ is an $i$-extension. Thus, by Corollary $2.9, R \subset T$ satisfies GD, and so by Lemma $2.2, R \subset T$ is mated.

CoRollary 2.11. $R$ is an i-domain if and only if $R$ is mated.

Proposition 2.12. Let $R$ be GD. If $R \subset T$ is an i-extension, then $T$ is $G D$.

Proof. By [6, Theorem 1], it suffices to show that $T \subset V$ satisfies GD for each valuation overring $V$ of $T$. Let $P \subsetneq M$ be primes of $T$ and $N$ a prime of $V$ contracting to $M$. By the hypotheses, there exists a prime $Q \subsetneq N$ such that $Q \cap R=P \cap R$. Thus $Q \cap T$ and $P$ have the same contraction in $R$, and so are equal.

COROLlARY 2.13. If $R$ is an i-domain, then every overring of $R$ is GD.

Proof. $R$ is GD by Corollary 2.9. Now apply Proposition 2.12.

Proposition 2.14. $R$ is an i-domain if and only if $R \subset \bar{R}$ is an i-extension and $\bar{R}$ is Prüfer.

Proof. In case $R=\bar{R}$, the result is immediate from [11, Theorem 16.10]. 
So suppose $R \subsetneq \bar{R}$. The "only if" direction is clear. For the "if" direction, we suppose that $R$ is not an $i$-domain. Thus, there exists an overring $T$ of $R$ and distinct primes $P$ and $Q$ of $T$ such that $P \cap R=Q \cap R$. Consider the following diagram

$$
\begin{aligned}
& \bar{R} \subset \bar{R} T \\
& \cup \quad \cup \\
& R \subset T .
\end{aligned}
$$

Since $T \subset \bar{R} T$ is an integral extension, there exist distinct primes $P^{\prime}$ and $Q^{\prime}$ in $\bar{R} T$ such that $P^{\prime} \cap T=P$ and $Q^{\prime} \cap T=Q$. Now $R \subset \bar{R} T$, as the composite of the $i$-extensions $R \subset \bar{R}$ and $\bar{R} \subset \bar{R} T$, is an $i$-extension, contradicting $P^{\prime} \cap R=$ $Q^{\prime} \cap R$, to complete the proof.

COROLlaRY 2.15. $R$ is a local $i$-domain if and only if $\bar{R}$ is a valuation ring.

Proof. Assume $R$ is a local $i$-domain. Then $\bar{R}$ is an integrally closed $i$-domain, local by integrality; i.e., $\bar{R}$ is a valuation ring.

Conversely, assume $\bar{R}$ is a valuation ring. Then $R$ is local, again by integrality. By Proposition 2.14, it now suffices to show that $R \subset \bar{R}$ is an $i$-extension. This follows since $\bar{R}$ is local treed and $R \subset \bar{R}$, being integral, satisfies INC.

We pause to remark that Corollaries 2.13 and 2.15 combine to recover [7, Corollary 2.5].

COROllary 2.16. Let $R$ be a Noetherian domain. Then $R$ is an i-domain if and only if $R \subset \bar{R}$ is an i-extension and $\operatorname{dim}(R) \leqslant 1$.

Proof. Assume $R$ is an $i$-domain. Then, by Corollary 2.9, $R$ is GD, so that [4, Proposition 7] gives $\operatorname{dim}(R) \leqslant 1$.

The converse is attained from Proposition 2.14 by showing that $\bar{R}$ is Dedekind. For this, we make a direct appeal to [18, Exercise 13, p. 73].

EXAMPLE 2.17. This example will show that the requirement of $R \subset \bar{R}$ being an $i$-extension is needed in Corollary 2.16 and hence in Proposition 2.14. Let $C$ be the complex numbers, and $x$ and $y$ indeterminates over $C$. Let $R$ be the localization of $C[x, y] /\left(y^{2}-x^{3}-x^{2}\right)$ at the image of $(x, y)$. Then $R$ is a local Noetherian domain, $\operatorname{dim}(R)=1$, and $\bar{R}$ possesses exactly two maximal ideals. (In particular, this example shows that an extension satisfying GD need not be an $i$-extension.)

In [6, Theorem 1], it was shown that valuation overrings of $R$ serve as test extension rings in determining whether $R$ is GD. Example 2.17 shows that valuation overrings of $R$ are, in general, not enough to test whether $R$ is an $i$-domain. However, we do have the following proposition.

Proposition 2.18. $R$ is an i-domain if and only if $R \subset V$ is an i-extension for each valuation overring $V$ of $R$ and $R \subset \bar{R}$ is an i-extension. 
Proof. The "only if" half is trivial. To complete the proof it suffices, by Proposition 2.14 , to show that $\bar{R}$ is Prüfer. The valuation overrings of $R$ coincide with those of $\bar{R}$; so $\bar{R} \subset V$ is an i-extension (since $R \subset V$ is) for each valuation overring $V$ of $\bar{R}$. Then [11, Theorem 16.10] implies that $\bar{R}$ is Prufer.

In [7] , Dobbs studies ascent and descent of GD domains for integral extensions. We next consider some similar questions for $i$-domains.

Proposition 2.19 (Ascent). Let $R$ be an i-domain with $\mathrm{gf}(R)=K$, and let $L$ be an algebraic extension field of $K$. Then $R^{*}$, the integral closure of $R$ in $L$, is a Prüfer domain.

Proof. Since $R$ is an $i$-domain, Proposition 2.14 implies that $\bar{R}$ is Prüfer. But $R^{*}$ is the integral closure of $\bar{R}$ in $L$, so that Prüfer's ascent result [11, Theorem 18.3] completes the proof.

An example of Heinzer-Ohm, summarized in [7, Example 2.1], shows that, without further assumptions, descent of $i$-domains fails for integral extensions.

Proposition 2.20(DESCENT). Let $T$ be an i-domain which is integral over $R$. If $T$ is local or $R$ is integrally closed, then $R$ is an $i$-domain.

Proof. First, assume that $T$ is a local $i$-domain, and let $S$ be any overring of $R$. Then, as in Proposition 2.14, we focus our attention on the following diagram:

$$
\begin{aligned}
& T \subset T S \\
& \cup \quad U \\
& R \subset S
\end{aligned}
$$

$R \subset T$ is an $i$-extension since it satisfies INC (by integrality) and $T$ is local treed, while $T \subset T S$ is an $i$-extension since $T$ is an $i$-domain and $T S \subset \operatorname{qf}(T)$. Thus, $R \subset T S$ is an $i$-extension. However, $S \subset T S$ satisfies LO, which implies that $R \subset S$ is an $i$-extension, and completes this part of the proof.

Now, suppose that $R$ is integrally closed. From Proposition 2.14 , we have that $\bar{T}$ is Prüfer, and [11, Theorem 18.4] implies that $R$ is Prüfer, thus finishing the proof.

Until this point, we have given no explicit examples of $i$-domains other than Prüfer domains. We now develop the device which will give rise (in Proposition 2.23) to nonintegrally closed $i$-domains. We list the following results without proof, as their proofs are straightforward applications of [11, Theorem $\mathbf{A}$ (c), (d), (e), p. 560] and [1, Theorem 3.1].

LEMMA 2.21. Let $V$ be a valuation ring of the form $F+M$, where $F$ is a field and $M$ is the maximal ideal of $V$. Let $R \subset T$ be subrings of $F$. Then 
$R \subset T$ is an i-extension if and only if $R+M \subset T+M$ is an i-extension.

Proposition 2.22. Let $V$ be a valuation ring of the form $F+M$, and let $R$ be a subring of $F$. Then $R+M$ is an i-domain if and only if $R \subset T$ is an i-extension for each ring $T$ satisfying $R \subset T \subset F$.

Proposition 2.23. Let $V$ be a valuation ring of the form $F+M$, and let $K$ be a subfield of $F$. Then $K+M$ is an i-domain if and only if $F$ is algebraic over $K$.

In the pursuit of GD domains from a topological point of view, $i$-domains were a natural point of departure. In view of Proposition 2.14 it is next of interest to study domains whose integral closures are Prüfer.

A domain $R$ is called an inc-domain if $R \subset T$ satisfies INC for each overring $T$ of $R$. Many of the lemmas and propositions for $i$-extensions and $i$-domains have immediate inc analogues, and for reasons of space we do not include them here.

Lemma 2.24. Let $R$ be local and integrally closed. If $u \in \mathrm{qf}(R) \backslash R$ and $R \subset R[u]$ satisfies $I N C$, then $u^{-1} \in R$.

Proof. Assume $u^{-1} \notin R$. If $M$ is the maximal ideal of $R$, then Seidenberg's $\left(u, u^{-1}\right)$-lemma [18, Theorem 67] shows that $M R[u]$ is a nonmaximal prime of $R[u]$ which lies over $M$. Since any maximal ideal of $R[u]$ containing $M R[u]$ contracts to $M$, our INC assumption is contradicted.

COROLlaRY 2.25. Let $R$ be integrally closed. Then $R$ is an inc-domain if and only if $R$ is Prüfer.

Proof. Assume $R$ is an inc-domain. For each $P \in \operatorname{Spec}(R), R_{P}$ is then a local integrally closed inc-domain, hence a valuation ring (by Lemma 2.24), so that $R$ is Prüfer.

The other direction is clear since any $i$-domain (in particular, a Prüfer domain) is an inc-domain.

PROPOSITION 2.26. $R$ is an inc-domain if and only if $\bar{R}$ is Prüfer.

Proof. The "only if" part follows from Corollary 2.25. The converse follows readily from the "rectangle argument", as given in the proofs of Proposition 2.14 and Proposition 2.20, keeping in mind that integral extensions satisfy GU.

Corollary 2.27. Let $R$ be a Noetherian domain. Then $R$ is an inc-domain if and only if $\operatorname{dim}(R) \leqslant 1$.

Proof. Suppose $R$ is an inc-domain. Then $\bar{R}$ is a Krull domain [27, The- 
orem 33.10] which is, by Proposition 2.26, also Prüfer. Hence, [11, Theorem 35.16] gives that $\operatorname{dim}(\bar{R}) \leqslant 1$, and thus $\operatorname{dim}(R) \leqslant 1$.

If $\operatorname{dim}(R)=1$ then, by the Krull-Akizuki theorem [18, Theorem 93], each overring of $R$ has dimension at most 1 , whence $R$ is an inc-domain.

We next give an example of an inc-domain which is not treed (and which, therefore, is not GD). This pathology will not lead us to abandon interest in inc-domains for questions about GD. Indeed, inc-domains will play an important role in $\$ 4$.

EXAMPLE 2.28. We next present an example of a domain $R$ such that $R$ is not treed, $\bar{R}$ is Prüfer, and each proper overring of $R$ contains $\bar{R}$. In [10], Gilmer and Heinzer say that $R$ has a unique minimal overring $R_{1}$ if $R \subsetneq R_{1}$ and if any overring of $R$, other than $R$ itself, contains $R_{1}$. In our example, $\bar{R}$ will be the unique minimal overring of $R$. The example is a slight modification of [10, Example 4.3], with whose details the reader is assumed to be familiar.

Let $k$ be a field, $x$ and $y$ indeterminates over $k$, and $K=k(x, y)$. Define a rank 2 valuation ring $W$ of $K$ over $k$ with value group $\mathbf{Z} \oplus \mathbf{Z}$ ordered lexicographically by sending $x$ and $y$ to $(0,1)$ and $(1,0)$, respectively, and then taking the value of a polynomial in $k[x, y]$ to be the infimum of the values of the monomials occurring in that polynomial. Similarly define a rank 2 valuation ring $V$ by sending $x$ and $y$ to $(1,0)$ and $(0,1)$ respectively, etc. Observe that $W=$ $k+x W$ and $V=k+y V$. It is easy to see that $\operatorname{Spec}(W) \cap \operatorname{Spec}(V)=\{0\}$, so that the prime spectrum of $S=V \cap W$ looks like

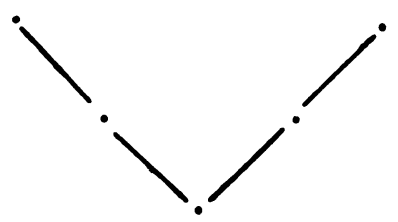

as a poset. Now by [20, Theorem 2.5], $R=k+J(S)$ is a local domain with spectrum looking like

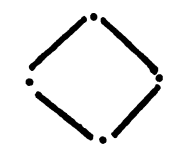

as a poset. By reasoning as in [10], one may verify that this $R$ has the properties promised above.

The remainder of this section continues the study of inc- and $i$-domains, and builds machinery needed for the remaining sections.

Proposition 2.29. $R$ is an inc-domain if and only if $R \subset V$ is an inc-extension for each valuation overring $V$ of $R$. 
Proof. Let $R \subset V$ satisfy INC for all valuation overrings $V$ of $R$. Then clearly $\bar{R} \subset V$ satisfies INC for all valuation overrings $V$ of $\bar{R}$; [11, Theorem 16.10], in conjunction with Proposition 2.26, completes this direction. The other direction is obvious.

REMARK 2.30. Throughout this paper, we shall be alert to the theme suggested by [5, Proposition 3.2], [6, Theorem 1] and Proposition 2.29; that is, we shall attempt to establish sufficiency of valuation overrings as test extensions for each class of domains considered.

The relationship between inc- and $i$-domains is easy to determine and is given below.

Proposition 2.31. $R$ is an i-domain if and only if $R$ is an inc-domain and $R \subset \bar{R}$ is an i-extension.

Proof. Since one half is obvious, we assume that $R$ is an inc-domain and $R \subset \bar{R}$ an $i$-extension. First, apply Proposition 2.26 to get $\bar{R}$ Prüfer; then use Proposition 2.14 to complete the proof.

We next present a variant of Proposition 2.12.

Proposition 2.32. Let $R$ be GD. If $T$ is treed and $R \subset T$ satisfies $I N C$, then $T$ is $G D$.

Proof. It suffices to establish that $T \subset V$ satisfies GD for each valuation overring $V$ of $T$. Let $P \subsetneq Q$ be primes in $T$, and $M$ a prime of $V$ lying over $Q$. Since $R$ is GD, there exists a prime $N$ of $V$ with $N \subsetneq M$ and $N \cap R=P \cap R$. As $T$ is treed, $N \cap T$ and $P$ are comparable, hence equal (by INC), to complete the proof.

The final aims of this section are to prove a lemma which will figure largely in $\S 4$ and to infer a companion for Corollary 2.15 .

LEMma 2.33. If each overring of $R$ is semilocal, then $R$ is an inc-domain.

Proof. Deny. Then there exists an overring $T$ of $R$ with primes $Q_{1} \subsetneq Q_{2}$ of $T$ such that $Q_{1} \cap R=Q_{2} \cap R \quad(=P)$. By passing to $R_{P} \subset T_{R \backslash P}$, we may assume $R$ is local with maximal ideal $P$. Let $u \in Q_{2} \backslash Q_{1}$. Observe that $R \subset R[u]$ fails to satisfy INC. Let $\phi: R[x] \rightarrow R[u]$ be the $R$-algebra homomorphism sending $x$ to $u$; let $N_{1}=\phi^{-1}\left(Q_{1} \cap R[u]\right)$ and $N_{2}=\phi^{-1}\left(Q_{2} \cap R[u]\right)$. Then $P R[x]$ $\subset N_{1} \subsetneq N_{2}$ since $P R[u] \subset Q_{1} \cap R[u]$; moreover, $N_{1} \cap R=P=N_{2} \cap R$. By [23, Theorem 1], $N_{1}=P R[x]$, so that $\operatorname{ker}(\phi) \subset P R[x]$. Using the locality of $R$ and applying [23, Theorem 1] again, we conclude that, if $N \in \operatorname{Spec}(R[x])$ and $N \supsetneq P R[x]$, then $\phi(N)$ is a maximal ideal of $R[u]$ which lies over $P$. However, $R[x]$ has infinitely many primes $N \supsetneq P R[x]$, thus giving rise to infinitely many maximal ideals of $R[u]$ and contradicting the semilocality of $R[u]$. 
Proposition 2.34. $R$ is a local $i$-domain if and only if every overring of $R$ is local.

Proof. Let $R$ be a local $i$-domain. If an overring $T$ of $R$ has maximal ideals $M$ and $N$ then, since $R$ is local treed, we may assume without loss of generality that $M \cap R \subset N \cap R$. However, $R$ being (GD and) an $i$-domain gives $M \subset N$, whence $M=N$.

For the converse, combine Lemma 2.33 with Proposition 2.26 and Corollary 2.15 .

3. Open domains. A domain $R$ will be called open if $R \subset T$ is an open extension for each overring $T$ of $R$; call $R$ propen (standing for properly open) if $R \subset T$ is an open extension for each overring $T$ of $R$ satisfying $R \subset T \subsetneq \mathrm{qf}(R)$. Since open extensions satisfy GD, any propen domain is GD. Although open domains are necessarily propen, Example 5.11 shows that the converse fails in general. $\$ 5$ is devoted to studying those domains $R$ that are propen but not open. In this section, we investigate many facets of open domains, as well as giving several necessary and sufficient conditions for a domain to be open.

For $P, P^{\prime} \in \operatorname{Spec}(R)$, let $\left[P, P^{\prime}\right]=\left\{Q \in \operatorname{Spec}(R): P \subset Q \subset P^{\prime}\right\}$. If $M$ is a maximal ideal of $R$, we call $[0, M]$ a branch of $R$. A typical basic open set of $X=\operatorname{Spec}(R)$ is given, as usual, by $X_{r}=\{Q \in \operatorname{Spec}(R): r \notin Q\}$ where $r \in R$; a nonempty closed set in $X$ is then of the form $F=V(I)=\{Q \in \operatorname{Spec}(R): I \subset Q\}$ where $I$ is an ideal of $R$.

$R$ is called a $G$-domain [18, pp. 12-13], if the nonzero prime ideals of $R$ have nonzero intersection, equivalently if $\mathrm{qf}(R)$ is finitely generated over $R$. It follows easily that $R$ is a $G$-domain if and only if $\{0\}$ is open in $\operatorname{Spec}(R)$, a characterization that will serve our purposes below.

Lemma 3.1. Let $P \in \operatorname{Spec}(R)$. Then, $R \subset R_{P}$ is open if and only if $[0, P]$ is an open set in $\operatorname{Spec}(R)$.

Proposition 3.2. The following are equivalent on $R$ :

(a) $R$ is open.

(b) $R$ is a propen $G$-domain.

(c) $R$ is $G D$ and $[0, P]$ is open for each $P \in \operatorname{Spec}(R)$.

Proof. The characterization of $G$-domains stated above shows that (a) $\Leftrightarrow$ (b). By Lemma 3.1, (a) $\Rightarrow$ (c). To prove that (c) $\Rightarrow$ (a), let $T$ be an overring of $R$ and $t$ an element of $T$; set $X=\operatorname{Spec}(T)$ and consider $f: X \rightarrow \operatorname{Spec}(R)$. Observe that $X_{t}=\bigcup_{\alpha \in \Omega}\left[0, Q_{\alpha}\right]$ for some set $\Omega$. The GD assumption in (c) implies that $f\left(X_{t}\right)=\bigcup_{\alpha \in \Omega}\left[0, Q_{\alpha} \cap R\right]$ which is, by the second condition in (c), open in $\operatorname{Spec}(R)$. Hence $(\mathrm{c}) \Rightarrow(\mathrm{a})$, completing the proof. 
PRoposition 3.3. If $R \subset R_{M}$ is open for each maximal ideal $M$ of $R$, then $R$ is semilocal.

Proof. Let $\left\{M_{i}: i \in I\right\}$ be the set of maximal ideals of $R$. By Lemma 3.1, each $\left[0, M_{i}\right]$ is open in $\operatorname{Spec}(R)$, so that $\left\{\left[0, M_{i}\right]: i \in I\right\}$ gives an open cover of $\operatorname{Spec}(R)$. As $\operatorname{Spec}(R)$ is (quasi-) compact [2, Proposition 12, p. 101], there is a finite subcover, indexed by a (finite) subset $J$ of $I$. For each $i \in I$, there exists $j \in J$ with $M_{i} \in\left[0, M_{j}\right]$, so that $M_{i}=M_{j}$, to complete the proof.

COROLlaRY 3.4. If $R$ is propen, then $R$ is semilocal.

COROLLARY 3.5. If $R$ is propen not open, then $\operatorname{dim}(R)$ is infinite.

Proof. Deny. Since $R$ is treed, Corollary 3.4 implies that $\operatorname{Spec}(R)$ is a finite set. Then [18, Exercise 3, p. 19] shows that $R$ is a $G$-domain, contradicting Proposition 3.2.

For a proof of Corollary 3.5 which does not rely on Corollary 3.4, again we deny, and let $n=\operatorname{dim}(R)$. Since $R$ is not open, $R \neq \mathrm{qf}(R)$; finiteness of $n$ therefore permits us to select a height 1 prime $P$ of $R$. Note that $\left\{0 R_{P}\right\}$ is open in $\operatorname{Spec}\left(R_{P}\right)$, although $\{0\}$ is not open in $\operatorname{Spec}(R)$, thus contradicting the assumption that $R \subset R_{P}$ is open.

Corollary 3.6. Let $\operatorname{dim}(R)<\infty$. Then $R$ is open if and only if $R$ is propen.

Proof. If $R$ is propen, an appeal to Corollary 3.5 gives that $R$ is open. The converse is trivial.

REMARK 3.7. Numerous examples of propen not open domains, as well as related theory, will appear in $\S 5$.

In view of Proposition 3.2, it is of some interest to investigate $G$-domains further. In [18, §1.3], many basic facts about $G$-domains are established; we now present other pertinent information.

LEMmA 3.8. Let $R \subset T$ be an open extension. If $T$ is a G-domain, then $R$ is a G-domain.

Proof. $\{0\}$ is open in $\operatorname{Spec}(T)$ since $T$ is a $G$-domain. The contraction map $f: \operatorname{Spec}(T) \rightarrow \operatorname{Spec}(R)$ sends $\{0\}$ to $\{0\}$. Thus $\{0\}$ is open in $\operatorname{Spec}(R)$, since $f$ is an open map, whence $R$ is a $G$-domain.

Lemma 3.9. Let $T$ be integral over $R$. If $T$ is a G-domain, then $R$ is a G-domain.

Proof. Assume that $T$ is a $G$-domain. As integrality implies that $f$ : $\operatorname{Spec}(T) \rightarrow \operatorname{Spec}(R)$ is a closed map, $f(\operatorname{Spec}(T) \backslash\{0\})$ is closed in $\operatorname{Spec}(R)$. How- 
ever, integrality also implies that $f$ is surjective and that $f^{-1}(0)=\{0\}$, so that $\operatorname{Spec}(R) \backslash\{0\}=f(\operatorname{Spec}(T) \backslash\{0\})$. Hence $\{0\}$ is open in $\operatorname{Spec}(R)$, and this completes the proof.

We now return to open domains. The next result is in the spirit of Remark 2.30.

PROPOSITION 3.10. The following three conditions on $R$ are equivalent:

(a) $R$ is open.

(b) $R \subset V$ is open for each valuation overring $V$ of $R$.

(c) $R \subset T$ is open for each integral domain $T$ containing $R$.

Proof. (a) $\Rightarrow$ (b) and (c) $\Rightarrow$ (a) are trivial.

Assume (b). By [6, Theorem 1], $R$ is GD. In order to establish (a), Propositions 3.1 and 3.2 show that it suffices to prove $R \subset R_{P}$ is open for each $P \in \operatorname{Spec}(R)$. If $V$ is chosen as a dominating valuation overring of $R_{P}$, consider $f: \operatorname{Spec}(V) \rightarrow \operatorname{Spec}\left(R_{P}\right)$ and $g: \operatorname{Spec}\left(R_{P}\right) \rightarrow \operatorname{Spec}(R)$. As $R_{P} \subset V$ satisfies GD, $f$ is surjective. Since $g f$ is open by assumption, it is clear (cf. [8, Exercise 7(b), p. 96]) that $g$ is open. Hence, (b) $\Rightarrow$ (a).

Finally, assume (a), and let $T$ be a domain containing $R$. Since $R \subset T$ satisfies GD (cf. [6, Theorem 1]), the argument which was used to establish the last part of Proposition 3.2 now shows that $R \subset T$ is open. Thus, (a) $\Rightarrow$ (c), to complete the proof.

Corollary 3.11. Let $R \subset T$ be an i-extension. If $R$ is open, then $T$ is open.

Proof. Let $S$ be an overring of $T$, and consider $f: \operatorname{Spec}(S) \rightarrow \operatorname{Spec}(T)$ and $g: \operatorname{Spec}(T) \rightarrow \operatorname{Spec}(R)$. As $g f$ is open and $g$ is injective, it is clear (cf. [8, Exercise 7(c), p. 96]) that $f$ is open, which completes the proof.

Remark 3.12. In [6, Theorem 1], the GD analogue of Proposition 3.10 was established. Moreover, it was shown there that $R$ being GD is equivalent to $R \subset R[u]$ satisfying GD for each $u$ in $\mathrm{qf}(R)$. A combination of Corollary 2.8 and Proposition 3.3 shows that the open analogue of this last equivalence is false: for a specific counterexample, choose any nonsemilocal Prüfer domain. It will be shown later that this analogue fails even in the semilocal case.

Before proving the next important lemma, we introduce some terminology. We say that a prime $P$ of $R$ is proper minimal over a prime $Q$ of $R$ if $Q \subsetneq P$ and $[Q, P]=\{Q, P\}$.

LEMMA 3.13. Let $R$ be semilocal treed, with maximal ideals $M_{1}, \ldots, M_{t}$. Let $P \in \operatorname{Spec}(R)$. Then $[0, P]$ is open if and only if one of the following two conditions holds:

(a) $P$ is not maximal and: for each $1 \leqslant j \leqslant t$, there exists a unique prime 
$Q_{j} \subset M_{j}$ such that $Q_{j}$ is proper minimal over $\bigcup\left\{Q \in \operatorname{Spec} R: Q \subset P \cap M_{j}\right\}$.

(b) $P$ is maximal, say $M_{i}$ and: for each $j \neq i$, there exists a unique prime $Q_{j} \subset M_{j}$ such that $Q_{j}$ is proper minimal over $\bigcup\left\{Q \in \operatorname{Spec}(R): Q \subset M_{i} \cap M_{j}\right\}$.

Proof. Assume (a). We claim that

$$
[0, P]^{\prime}=\bigcup_{i=1}^{t} V\left(Q_{i}\right)
$$

where $[0, P]$ ' denotes $\operatorname{Spec}(R) \backslash[0, P]$.

Indeed, let $N \in[0, P]^{\prime} ;$ select $j$ such that $N \subset M_{j}$. If $Q_{j} \not N$ then, since $R$ is treed, $N \subsetneq Q_{j}$; since $N \not \subset P$, minimality of $Q_{j}$ gives $\bigcup\left\{Q: Q \subset P \cap M_{j}\right\} \not \subset N$, whence $N \subsetneq \bigcup\left\{Q: Q \subset P \cap M_{j}\right\}$ and $N \subset P$, a contradiction. Hence $Q_{j} \subset N$ and this puts $N$ in $\bigcup_{i=1}^{t} V\left(Q_{i}\right)$.

To prove the reverse inclusion, let $N \in \bigcup_{i=1}^{t} V\left(Q_{i}\right)$; that is, for some $j$, $Q_{j} \subset N$. If $N \notin[0, P]^{\prime}$, then $N \subset P$, so that $Q_{j} \subset P$, contradicting the fact that $Q_{j}$ properly contains $\bigcup\left\{Q: Q \subset P \cap M_{j}\right\}$. Therefore $N \in[0, P]^{\prime}$ and the claim is established. Then $[0, P]$, as the complement of a finite union of closed sets, is certainly open.

Next, assume (b); without loss of generality, take $P=M_{1}$. We claim that $[0, P]^{\prime}=\bigcup_{i=2}^{t} V\left(Q_{i}\right)$. Indeed, if $N \in[0, P]^{\prime}$, then $N \not \subset P$ and $N \subset M_{j}$ for some $j \neq 1$; by arguing as above, we get $Q_{j} \subset N$, whence $N \in \bigcup_{i=2}^{t} V\left(Q_{i}\right)$. The reverse inclusion is also obtained by aping the earlier argument.

Conversely, assume $[0, P]$ is open and $P$ is not maximal. Now, there exists a nonzero ideal $I$ of $R$ such that $[0, P]^{\prime}=V(I)$. Fix $i, 1 \leqslant i \leqslant t$. Since $P$ is not maximal, $I \subset M_{i}$; let $Q_{i}$ be the intersection of the primes which contain $I$ and are contained in $M_{i}$. Note that $Q_{i}$ is prime since $R$ is treed. We now show that $Q_{i}$ is the unique prime contained in $M_{i}$ and proper minimal over $\bigcup\{Q: Q \subset P \cap$ $\left.M_{i}\right\}$. Observe that $\bigcup\left\{Q: Q \subset P \cap M_{i}\right\} \subsetneq Q_{i}$; for if not, then since $R$ is treed, $Q_{i} \subset \bigcup\left\{Q: Q \subset P \cap M_{i}\right\}$ and $Q_{i} \subset P$, contradicting $I \subset Q_{i}$. Next, to establish minimality, let $N$ be a prime such that $\bigcup\left\{Q: Q \subset P \cap M_{i}\right\} \subsetneq N \subset Q_{i}$. Since $N$ properly contains the union, $N \not \subset P$. Thus $N \in[0, P]^{\prime}=V(I)$; that is, $I \subset N$, and so $Q_{i}=N$ by the construction of $Q_{i}$. The uniqueness asserted of $Q_{i}$ in (a) is immediate since $R$ is treed.

Now assume $[0, P]$ is open and $P$ is maximal. Without loss of generality, $P=M_{1}$ and $t \geqslant 2$. The openness of $[0, P]$ implies that there exists a nonzero ideal $I$ of $R$ such that $[0, P]^{\prime}=V(I)$. Thus $I \subset M_{j}$ for each $j \neq 1$. As before, for each $j \neq 1$, let $Q_{j}$ be the intersection of the primes containing $I$ and contained in $M_{j}$. By arguing as above, we see that $Q_{j}$ has the properties asserted in (b), to complete the proof.

Proposition 3.14. $R$ is treed and $[0, P]$ is open for each $P \in \operatorname{Spec}(R)$ if and only if $R$ is semilocal and each branch of $R$ is well-ordered under inclusion. 
Proof. Suppose that $R$ is treed and each $[0, P]$ is open. That $R$ is semilocal we have shown before (Proposition 3.3); let $M_{1}, \ldots, M_{t}$ be the maximal ideals of $R$. If, for some $1 \leqslant i \leqslant t,\left[0, M_{i}\right]$ is not well-ordered by inclusion, then there exists a nonempty subset $\left\{Q_{\alpha}: \alpha \in \Omega\right\}$ of $\left[0, M_{i}\right]$ with no first element. Let $P=\bigcap_{\alpha \in \Omega} Q_{\alpha}$, and note that $P \subsetneq M_{i}$. Now, by Lemma 3.13, there exists a unique prime $Q_{i} \subset M_{i}$ such that $Q_{i}$ is proper minimal over $\cup\{Q: Q \subset$ $\left.P \cap M_{i}\right\}=P$. Hence $Q_{i} \not \subset P$, so that there exists $\beta \in \Omega$ with $Q_{i} \not \subset Q_{\beta}$. Since $R$ is treed, $Q_{\beta} \subsetneq Q_{i}$, whence $P \subset Q_{\beta} \subsetneq Q_{i}$. The proper minimality of $Q_{i}$ over $P$ gives that $P=Q_{\beta}$, contradicting the condition that $\left\{Q_{\alpha}: \alpha \in \Omega\right\}$ have no first element.

For the converse, assume $R$ is semilocal with well-ordered branches. It is clear that $R$ is treed. Let $P \in \operatorname{Spec}(R)$. To show that $[0, P]$ is open, we consider two cases. As above, let $M_{1}, \ldots, M_{t}$ be the maximal ideals of $R$,

Case 1. $P$ a maximal ideal of $R$. Without loss of generality, $P=M_{1}$ and $R$ is not local. Fix $j \neq 1$. Let $N_{j}=\bigcup\left\{Q: Q \subset M_{j} \cap P\right\}$ and $F_{j}=\left\{Q: N_{j} \subsetneq Q \subset\right.$ $\left.M_{j}\right\}$. Note that $F_{j} \neq \varnothing$ since $N_{j} \subsetneq M_{j}$. Thus, by hypothesis, $F_{j}$ has a least element, say $Q_{j}$. It is clear that $Q_{j}$ plays the role of the (necessary unique) object described in condition (b) of Lemma 3.13. Thus, by Lemma 3.13, $[0, P]$ is open.

Case 2. $P$ not maximal. For each $1 \leqslant i \leqslant t$, define $N_{i}$ and $F_{i}$ as before, use the well-ordering hypothesis to produce $Q_{i}$, satisfying condition (a) of Lemma 3.13 , to complete the proof.

Corollary 3.15. Let $R$ be a semilocal domain each of whose branches is well-ordered under inclusion. If $R \subset T$ satisfies $G D$, then $R \subset T$ is an open extension.

Proof. Let $X=\operatorname{Spec}(T)$, let $r \in R$, and consider $f: X \rightarrow \operatorname{Spec}(R)$. Arguing as in Proposition 3.2 gives $f\left(X_{r}\right)=\bigcup_{\alpha \in \Omega}\left[0, P_{\alpha}\right]$ for some collection of primes $P_{\alpha}$ of $R$. A direct appeal to Proposition 3.14 shows that $\left[0, P_{\alpha}\right]$ is open for each $\alpha \in \Omega$, so that $f\left(X_{r}\right)$ is open in $\operatorname{Spec}(R)$. Thus, $f$ is open.

THEOREM 3.16. $R$ is open if and only if $R$ is GD and semilocal and each branch of $R$ is well-ordered under inclusion.

Proof. Assume $R$ is open. Then $R$ is clearly GD, and Proposition 3.3 shows $R$ is semilocal. That the branches of $R$ are each well-ordered under inclusion, follows from Propositions 3.2 and 3.14.

The converse is an immediate consequence of Corollary 3.15 .

COROLLARY 3.17. The following are equivalent on $R$ :

(a).$R$ is open.

(b) $R_{P}$ is open for each $P \in \operatorname{Spec}(R)$ and $R$ is semilocal.

(c) $R_{M}$ is open for each maximal ideal $M$ of $R$ and $R$ is semilocal. 
Proof. Corollary 3.11 and Proposition 3.3 combine to yield (a) $\Rightarrow(b)$, while (b) $\Rightarrow$ (c) trivially. To show (c) $\Rightarrow$ (a), assume (c). It is evident (cf. [5, Lemma 2.1]) that $R$ is GD. By Theorem 3.16, it is enough to prove that $[0, M]$ is well-ordered under inclusion for each maximal ideal $M$ of $R$. Now, the openness of $R_{M}$ gives that $\left[0, M R_{M}\right]$ is well-ordered, so that $[0, M]$ is indeed wellordered, to complete the proof.

If we remove the semilocality condition, the preceding corollary fails, as the ring of integers so aptly shows. However, we do have the following corollary in that case.

COROLLARY 3.18. The following are equivalent on $R$ :

(a) $R$ is GD and each branch of $R$ is well-ordered under inclusion.

(b) $R_{P}$ is open for each $P \in \operatorname{Spec}(R)$.

(c) $R_{M}$ is open for each maximal ideal $M$ of $R$.

Proof. (b) $\Rightarrow$ (c) is trivial, while part of the proof of Corollary 3.17 gives (c) $\Rightarrow$ (a). Finally, assume (a), and let $P \in \operatorname{Spec}(R)$. Then $R_{P}$ is GD; since $[0, P]$ is well-ordered under inclusion, so is $\operatorname{Spec}\left(R_{P}\right)$. Applying Theorem 3.16 yields (b), and completes the task.

Corollary 3.19. Let $P \in \operatorname{Spec}(R)$. If $R$ is open and $R / P$ is $G D$, then $R / P$ is open.

Proof. $R / P$ inherits from $R$ the properties of being semilocal and having each branch well-ordered under inclusion. Apply Theorem 3.16.

Proposition 3.20. The following are equivalent for any Noetherian domain $R$ :

(a) $R$ is semilocal and $\operatorname{dim}(R) \leqslant 1$.

(b) $R$ is open.

(c) $R$ is propen.

(d) $R$ is a G-domain.

(e) Every overring of $R$ is open.

(f) Every overring of $R$ is propen.

Proof. (a) $\Rightarrow$ (b) is immediate from Theorem 3.16 , and (b) $\Rightarrow$ (a) follows from Proposition 3.2 and [18, Theorem 146]. (b) $\Rightarrow$ (c) is trivial, and (c) $\Rightarrow$ (b) is a consequence of [4, Proposition 7] and Corollary 3.6 [18, Theorem 146], combined with Theorem 3.16, shows (d) $\Rightarrow(b)$; Proposition 3.2 gives (b) $\Rightarrow(d)$. Note that (e) $\Rightarrow(\mathrm{b})$ is obvious. To show (b) $\Rightarrow(\mathrm{e})$, let $S$ be an overring of $R$. Since $\operatorname{dim}(R) \leqslant 1$ by (b), the Krull-Akizuki theorem [18, Theorem 93] implies $S$ is Noetherian and $\operatorname{dim}(S) \leqslant 1$; from [18, Exercise 21, p. 64] , and the semilocality of $R, S$ is semilocal, hence open. Hence (b) $\Rightarrow(\mathrm{e})$. Next $(\mathrm{f}) \Rightarrow(\mathrm{e})$, since 
(c) $\Rightarrow$ (b) and all the rings involved are Noetherian. Finally, (e) $\Rightarrow(f)$ trivially, to complete the proof.

Motivated by Proposition 3.2 and Theorem 3.16, we present the following proposition.

Proposition 3.21. Let $R$ be semilocal. Then each branch of $R$ is wellordered under inclusion if and only if $R$ is treed and $R / P$ is a G-domain for each $P \in \operatorname{Spec}(R)$.

Proof. Assume that each branch of $R$ is well-ordered under inclusion. Clearly, $R$ is treed. If $P \in \operatorname{Spec}(R)$, then $R / P$ is semilocal and each branch of $R / P$ is well-ordered under inclusion; thus, we may reduce to the case $P=0$. Suppose $R$ is not a $G$-domain, and let $M_{1}, \ldots, M_{t}$ be the maximal ideals of $R$. Thus, there exists $j, 1 \leqslant j \leqslant t$, such that

$$
\bigcap\left\{Q \in \operatorname{Spec}(R): Q \neq 0 \text { and } Q \subset M_{j}\right\}=0 \text {, }
$$

contradicting the well-ordering of $\left[0, M_{j}\right]$.

For the converse, suppose that $[0, M]$ fails to be well-ordered under inclusion for some maximal ideal $M$ of $R$. Then some nonempty subset $Q_{\alpha}$ : $\alpha \in \Omega\}$ of $[0, M]$ has no first element. As the prime $P=\bigcap_{\alpha \in \Omega} Q_{\alpha}$ is not a member of $\left\{Q_{\alpha}: \alpha \in \Omega\right\}$, one checks readily that $R / P$ is not a $G$-domain, which completes the proof.

In $\S 2$, we considered questions related to the ascent and descent of $i$-domains for integral extensions. We now provide a similar analysis for open domains. Descent for open domains will be seen to be as well-behaved as for $i$-domains and GD domains. Ascent becomes more complicated, in part because an integral extension of a semilocal domain need not be semilocal.

Example 2.28 illustrates that, without further assumptions, descent of open domains fails for integral extensions; the Heinzer-Ohm example, reprised in [7, Example 2.1], shows the failure of ascent.

PROPOSITION 3.22. Let $R \subset T$ be an integral extension, where $R$ is $G D$ and $T$ is semilocal treed. Then $R$ is open if and only if $T$ is open.

Proof. By Proposition 2.32, $T$ is GD. If $R$ is open, INC readily implies that each branch of $T$ is well-ordered under inclusion, whence $T$ is open by Theorem 3.16 .

Conversely, assume $T$ is open. Integrality, together with the semilocality of $T$, gives that $R$ is semilocal. To complete the proof, we need, according to Theorem 3.16, only to show that each branch of $R$ is well-ordered under inclusion. Suppose, for some maximal ideal $M$ of $R$, that $[0, M]$ is not well-ordered under inclusion; that is, some nonempty subset $\left\{Q_{\alpha}: \alpha \in \Omega\right\}$ of $[0, M]$ has no 
first element. By LO, select $m \in \operatorname{Spec}(T)$ such that $m \cap R=M$. Let

$$
F=\left\{q \in \operatorname{Spec}(T): q \subset m \text { and } q \cap R \in\left\{Q_{\alpha}: \alpha \in \Omega\right\}\right\} ;
$$

note that $F \neq \varnothing$ since $R$ is GD. As $T$ is open, $F$ has a first element, say $q^{\prime}$. Since $q^{\prime} \cap R \in\left\{Q_{\alpha}: \alpha \in \Omega\right\}$, the supposed failure of well-ordering yields $\beta \in \Omega$ such that $Q_{\beta} \subsetneq q^{\prime} \cap R$. However, $R \subset T$ satisfies GD, thus producing a prime $q^{\prime \prime}$ of $T$ such that $q^{\prime \prime} \subsetneq q^{\prime}$ and $q^{\prime \prime} \cap R=Q_{\beta}$. This contradicts the minimality of $q^{\prime}$ in $F$, and completes the proof.

COROLlaRY 3.23. Let $T$ be finitely generated as a module over $R$, let $R$ be $G D$ and let $T$ be treed. Then $R$ is open if and only if $T$ is open.

Proof. This proof is identical to that of Proposition 3.22, once we have shown that $R$ being semilocal implies $T$ is semilocal. Since $T$ is module-finite over $R$, this follows from [2, Proposition 3, p. 329].

COROllary 3.24. Let $R$ be an i-domain. Then $R$ is open if and only if $\bar{R}$ is open.

Proof. Apply Corollary 2.13 and Proposition 3.22.

We now prove the open analogue of Proposition 2.20.

Proposition 3.25. Let $T$ be an open domain which is integral over $R$. If either $T$ is local or $R$ is integrally closed, then $R$ is open.

Proof. If $T$ is local, then [7, Theorem 2.4] implies that $R$ is GD, so that Proposition 3.22 applies to show that $R$ is open.

Now assume that $R$ is integrally closed, and let $S$ be any overring of $R$. To show $R$ is open, we use the now-familiar "rectangle argument". By [9, Proposition 1.2], $R \subset T$ is open; moreover, $T \subset T S$ is open since $T$ is open. Hence $R \subset T S$ is open, as openness is transitive. Now $S \subset T S$, being integral, satisfies LO, from which it is clear (cf. [8, Exercise 7(b), p. 96]) that $R \subset S$ is open. This completes the proof.

We now provide (Corollary 3.28) means for constructing local nonvaluation open domains.

LEMMA 3.26. Let $V$ be a valuation ring of the form $F+M$, where $F$ is a field and $M$ is a maximal ideal of $V$. Let $R \subset T$ be subrings of $F$. Then $R \subset T$ is open if and only if $R+M \subset T+M$ is open.

Proof. Throughout this proof, we freely use the structure theory of $\operatorname{Spec}(R+M)[11$, Theorem A (c), (d), (e), p. 560]. Denote the contractions by

$$
f: X=\operatorname{Spec}(T) \rightarrow \operatorname{Spec}(R)=Y
$$


and

$$
F: \underline{X}=\operatorname{Spec}(T+M) \rightarrow \operatorname{Spec}(R+M)=\underline{Y} .
$$

We consider several cases.

Assume that $R \subset T$ is open. We consider $F\left(X_{a}\right)$.

Case 1. $a=t \in T$. If $t=0$, then $\underline{X}_{t}=\varnothing$ and $F\left(X_{t}\right)=\varnothing$ is open; thus, without loss of generality, $t \neq 0$. Let $U=\left\{P \in X: P+M \in \underline{X}_{t}\right\}$. Since $U=X_{t}$ and $f$ is open, there is a nonempty subset $\Lambda$ of $R \backslash\{0\}$ such that $f\left(X_{t}\right)=\bigcup_{g \in \Lambda} Y_{g}$. We claim that $F\left(X_{t}\right)=\bigcup_{g \in \Lambda} \underline{Y}_{g}$.

By considering several subcases, the above equality is easily verified.

Case 2. $a=m \in M$. Then $F\left(\underline{X}_{a}\right)=\underline{Y}_{m}$ is open in $\underline{Y}$.

Case 3. $a=t+m$, with $0 \neq t \in T$ and $0 \neq m \in M$. Let $U=\{P \in X$ : $\left.P+M \in \underline{X}_{t+m}\right\}$. Again, $U=X_{t}$, producing nonempty $\Lambda \subset R \backslash\{0\}$ such that $f\left(X_{t}\right)=\bigcup_{g \in \Lambda} Y_{g}$. Using an analysis by subcases as in Case 1, we may conclude that $F\left(X_{t+m}\right)=\bigcup_{g \in \Lambda} \underline{Y}_{g+m}$.

Hence $R+M \subset T+M$ is open.

Conversely, assume that $R+M \subset T+M$ is open, and consider $f\left(X_{t}\right)$, where $t \in T$. Without loss of generality, $X_{t} \neq \varnothing$, so that $t \neq 0$. As $F$ is open, there exists nonempty $\Lambda \subset(R+M) \backslash\{0\}$ such that $F\left(X_{t}\right)=\bigcup_{g \in \Lambda} \underline{Y}_{g}$. Since $X_{t} \neq \varnothing$, we have $\Lambda \not \subset M$. Let $\Lambda^{\prime}=\{r \in R: r+m \in \Lambda$ for some $m \in M\}$; then $\varnothing \neq \Lambda^{\prime} \neq\{0\}$. To complete the proof, it suffices to show $f\left(X_{t}\right)=\bigcup_{r \in \Lambda^{\prime}} Y_{r} ;$ which is a straightforward calculation.

The following " $R+M$ " results follow by using the theory thus developed combined with [11, Theorem A (c), (d), (e), p. 560] and [1, Theorem 3.1].

Corollary 3.27. Let $V$ be a valuation ring of the form $F+M$. Let $R$ be a subring of $F$. Then $R+M$ is open if and only if both $R$ and $V$ are open.

COROLlary 3.28. Let $V$ be a valuation ring of the form $F+M$, and let $K$ be a subfield of $F$. Then $K+M$ is open if and only if $V$ is open.

COROLlaRY 3.29. Let $V$ be a valuation ring of the form $F+M$, and let $R$ be a subring of $F$. Then every overring of $R+M$ is open if and only if $V$ is open and every ring $T$ satisfying $R \subset T \subset F$ is open.

In the remainder of this section, we pursue some topologically defined classes of domains. While these domains need not be open (indeed, they need not even be semilocal), we shall see that they form, in the semilocal case, nothing more than the class of GD domains.

A domain $R$ is said to be simple open (write: $R$ is SO) in case $R \subset R[u$ ] is open for each $u \in \mathrm{qf}(R)$; say that $R$ is finite type open (write: $R$ is FTO) in case $R \subset T$ is open for each finitely generated overring $T$ of $R$. 
The GD analogues of the above notions have received attention. It follows from [25, Lemma 3] that FTGD $\Leftrightarrow$ GD; this was strengthened in [6, Theorem 1], where it was proved that SGD $\Leftrightarrow$ GD. Since open extensions satisfy GD, we conclude that

$$
\text { FTO } \Rightarrow \mathrm{SO} \Rightarrow \mathrm{GD} \text {. }
$$

As explained in Remark 3.12, it follows from Corollary 2.8 that $i$-domains are FTO, thus destroying any hope of proving that an arbitrary FTO domain is open. For the special case of (semi) local domains, Theorem 3.16 and Corollary 2.8 combine to show that FTO and open are still not equivalent. For a concrete example, take a valuation domain $V$ whose spectrum is not well-ordered under inclusion.

The following lemma may be deduced by using the techniques in [9] .

LEMma 3.30. Let $T$ be integral over $R$. The following are equivalent:

(a) $R \subset T$ is open.

(b) $R \subset S$ is open for each finitely generated $R$-subalgebra $S$ of $T$.

(c) $R \subset R[u]$ is open for each $u \in T$.

COROllary 3.31. Let $T$ be an integral overring of a coherent domain $R$. If $R \subset T$ satisfies $G D$, then $R \subset T$ is open.

Proof. By Lemma 3.30 and [24, Lemma 1], we may assume that there exists $t \in T$ with $T=R[t]$. Since $R$ is coherent and $T$ is a finitely generated $R$-submodule of $\mathrm{qf}(R), T$ is finitely presented as an $R$-module. Then [16, Proposition 1.4.7] shows that $T$ is finitely presented as an $R$-algebra, and, since $R \subset$ $T$ satisfies GD, [16, Corollaire 1.10.4] applies to complete the proof.

REMARK 3.32. In the spirit of Corollary 3.31 , it would be interesting to know, given commutative rings $R \subset T$ with $R$ coherent and $T$ finitely generated as an $R$-algebra such that $R \subset T$ satisfies GD, whether $\operatorname{Spec}(T) \rightarrow \operatorname{Spec}(R)$ must be open. An affirmative answer would generalize a well-known corollary of Chevalley's constructability theorem for Noetherian rings [22, Theorem 8, p. 48] . The special case, in which $R$ is an integrally closed domain and $T=R[t]$ for some $t \in \mathrm{qf}(R)$, follows from McAdam's observation that [31, $(11: 13)]$ forces $T$ to be finitely presented as an $R$-algebra. Of course, [4, Corollary 4] implies that, if $R$ is a coherent integrally closed domain such that $R \subset R[u]$ satisfies GD for each $u \in \mathrm{qf}(R)$, then $R$ is Prüfer and, hence, FTO.

PROPOSITION 3.33. $R \subset \bar{R}$ is open if and only if $R \subset T$ is open for each domain $T$ which is integral over $R$.

Proof. The "if" direction is immediate. Conversely, assume that $R \subset \bar{R}$ is open, and let $T$ be integral over $R$. Use the "rectangle argument". As $\bar{R} T$ is 
integral over $\bar{R}$, [9, Proposition 1.2] implies that $\bar{R} \subset \bar{R} T$ is open; thus, $R \subset \bar{R} T$ is open. Consider $f: \operatorname{Spec}(\bar{R} T) \rightarrow \operatorname{Spec}(T)$ and $g: \operatorname{Spec}(T) \rightarrow \operatorname{Spec}(R)$. Since $g f$ is open and $f$ is surjective, [8, Exercise 7(b), p. 96] shows $g$ is open, as required.

Proposition 3.34. If $R$ is an $i$-domain and $R \subset T \subset \bar{R}$, then $R \subset T$ is open.

Proof. Since $R$ is FTO, an application of Lemma 3.30 completes the proof.

COROLlaRY 3.35. If $T$ is a domain which is integral over an i-domain $R$, then $R \subset T$ is open.

Proof. Apply Propositions 3.33 and 3.34.

We end $\S 3$ by giving a characterization of semilocal FTO domains.

PROPOSITION 3.36. Let $T$ be finitely generated over a semilocal domain $R$. If $R$ is $G D$, then $R \subset T$ is open.

Proof. Since $R$ is semilocal treed, each ideal of $R$ has only finitely many primes of $R$ minimal over it. As $R \subset T$ satisfies GD, [25, Theorem 1] applies to complete the proof.

Corollary 3.37. Let $R$ be semilocal. The following are equivalent on $R$ :

(a) $R$ is FTO.

(b) $R$ is $S O$.

(c) $R$ is $G D$.

Proof. (a) $\Rightarrow$ (b) trivially, (b) $\Rightarrow$ (c) by applying [6, Theorem 1], and (c) $\Rightarrow$ (a) by Propsoition 3.36.

COROLLARY 3.38. The following are equivalent on $R$ :

(a) $R$ is $G D$.

(b) $R_{P}$ is FTO for each $P \in \operatorname{Spec}(R)$.

(c) $R_{P}$ is $S O$ for each $P \in \operatorname{Spec}(R)$.

Proof. (a) $\Rightarrow$ (b) by [5, Lemma 2.1] and Corollary 3.37; (b) $\Rightarrow$ (c) is trivial; (c) $\Rightarrow$ (b) by [6, Theorem 1] and [5, Lemma 2.1].

REMARK 3.39. Since SO $\Rightarrow$ GD, Corollary 3.38 shows that SO and FTO each localize. If one could prove, for instance, that SO globalizes, then Corollary 3.38 would imply the equivalence of GD and SO. At present, we have no counterexamples to the possible implications GD $\Rightarrow \mathrm{SO}$ and $\mathrm{SO} \Rightarrow$ FTO. Notice that, for a Noetherian domain, one can use [4, Proposition 7] and [22, Theorem 8 , p. 48] to show the equivalence of FTO, SO, and GD. 
REMARK 3.40. The major interest of this section has been in studying open domains, while $\S 5$ will deal mainly with propen not open domains. We take this opportunity to point out that the "closed" analogues of these classes turn out to be less interesting. To be more specific, say that a domain $R$ is closed (respectively, properly closed) if $\operatorname{Spec}(T) \rightarrow \operatorname{Spec}(R)$ is a closed map for each overring $T$ of $R$ (respectively, for each overring $T$ of $R$ which is distinct from $\mathrm{qf}(R)$ ).

Observation 1. $R$ is closed if and only if $R$ is a field.

Proof. The "if" half is trivial. Conversely, if $\operatorname{Spec}(\mathrm{qf}(R)) \rightarrow \operatorname{Spec}(R)$ is closed, then $\{0\}$ is a closed set of $\operatorname{Spec}(R)$, whence 0 is maximal ideal of $R$ and $R$ is a field.

Observation 2. $R$ is properly closed if and only if $R$ is local and $\operatorname{dim}(R) \leqslant 1$.

Proof. The "if" half is immediate. Conversely, let $R$ be properly closed. Without loss of generality, $R$ is not a field; select a nonzero prime ideal $P$ of $R$. As $\operatorname{Spec}\left(R_{P}\right) \rightarrow \operatorname{Spec}(R)$ is closed, $[0, P]$ is a closed subset of $\operatorname{Spec}(R)$, whence $\operatorname{Spec}(R)=\{0, P\}$, to complete the proof.

4. Local homeomorphism domains. The aim of the present section is to study, and to give a topological characterization (in Theorem 4.16) of, those domains all of whose overrings are open. In view of the varied nature of the examples given by Dobbs in [5, Corollary 4.4], one expects that a similar study of domains all of whose overrings are GD would not be as fruitful.

We shall continue to use the definitions and notation established in previous sections. Besides those, we introduce the following: Let $f: X \rightarrow Y$ be a continuous map of topological spaces. We call $f$ a local injection if each point $x \in X$ has an open neighborhood $U$ such that $f: U \rightarrow f(U)$ is an injection. As usual (cf. [21, p. 149]), call $f$ a local homeomorphism if each point $x \in X$ has an open neighborhood $U$ such that $f(U)$ is open and $f$ maps $U$ homeomorphically onto $f(U)$. Since any local homeomorphism must be an open map, it follows that $f$ is a local homeomorphism if and only if $f$ is open and $f$ is a local injection.

We call an extension $R \subset T$ of domains an LH-extension (respectively, an $L I$-extension) if $\operatorname{Spec}(T) \rightarrow \operatorname{Spec}(R)$ is a local homeomorphism (respectively, a local injection). A domain $R$ is said to be an $L H$-domain (respectively, an $L I$-domain) if $R \subset T$ is an $L H$-extension (respectively, an $L I$-extension) for each overring $T$ of $R$. Thus, $R$ is an LH-domain if and only if $R$ is an LI-domain and $R$ is open.

Given a contraction map $f: \operatorname{Spec}(T) \rightarrow \operatorname{Spec}(R)$ and $P \in \operatorname{Spec}(R)$, denote the set $f^{-1}(P)$, the fiber of $P$, by fib $T(P)$. We say that $R \subset T$ has finite fibers if $\operatorname{~fib}_{T}(P)$ is finite for each $P \in \operatorname{Spec}(R) ; R$ is said to have finite fibers if $R \subset T$ has finite fibers for each overring $T$ of $R$.

As in the previous sections, some of the results are straightforward, and are stated without proof. 
Lemma 4.1. Let $X$ and $Y$ be topological spaces. If $X$ is compact and $f$ : $X \rightarrow Y$ is a local injection, then, for any point $y \in Y, f^{-1}(y)$ is a finite set.

Corollary 4.2. Any LI-extension has finite fibers.

ProOF. Lemma 4.1 applies directly since prime spectra are compact.

Remark 4.3. Lemma 4.1 generalizes [21, Example 2.3, p. 151]

Proposition 4.4. If $R \subset T$ is an LI-extension, then $R \subset T$ is an incextension.

Proof. Deny. Hence there exist distinct primes $P \subset Q$ of $T$ such that $P \cap R=Q \cap R$. As $f: \operatorname{Spec}(T) \rightarrow \operatorname{Spec}(R)$ is a local injection, there exists an open set $U$ in $\operatorname{Spec}(T)$ such that $Q \in U$ and $f: U \rightarrow f(U)$ is injective. However, $Q \in U$ implies $P \in U$, contradicting the injectivity of $f$ on $U$.

Thus any LI-domain is an inc-domain. However, with the aid of Corollary 4.2 and [7, Example 2.1], it follows that the converse is not generally true. Moreover, Example 2.28 illustrates that an LI-domain need not be treed.

Proposition 4.5. If $R$ is an LI-domain and $T$ is an overring of $R$, then $T$ is an LI-domain.

Proof. Let $S$ be an overring of $T$ and $P \in \operatorname{Spec}(S)$; consider $f: \operatorname{Spec}(S)$ $\rightarrow \operatorname{Spec}(T)$ and $g: \operatorname{Spec}(T) \rightarrow \operatorname{Spec}(R)$. Since $R$ is an LI-domain, there exists an open set $U$ in $\operatorname{Spec}(S)$ such that $P \in U$ and $g f$ is injective on $U$. Thus, $f$ is injective on $U$, so that $T$ is an LI-domain.

Proposition 4.6. Let $R \subset T$ be an LI-extension. If $R$ is $G D$, then $T$ is GD.

Proof. By [6, Theorem 1], it suffices to show that $T \subset V$ satisfies GD for each valuation overring $V$ of $T$. Let $P \subsetneq Q$ be primes of $T$, and let $N$ be a prime of $V$ lying over $Q$. By Proposition 4.4, $P \cap R \subsetneq Q \cap R$. Since $R \subset V$ satisfies GD, there exists a prime $N^{\prime}$ of $V$ such that $N^{\prime} \subsetneq N$ and $N^{\prime} \cap R=P \cap R$. Thus $N^{\prime} \cap T \subsetneq Q$. Furthermore, there exists an open subset $U$ of $\operatorname{Spec}(T)$ such that $Q \in U$ and $f: U \rightarrow \operatorname{Spec}(R)$ is injective. As $N^{\prime} \cap T$ and $P$ are members of $U$ with the same contraction in $R$, we have $N^{\prime} \cap T=P$, so that $T$ is GD.

Before establishing the promised characterization of domains all of whose overrings are open, we continue to develop needed machinery.

LEMMA 4.7. If $R$ has finite fibers, then each overring of $R$ has finite fibers.

Proposition 4.8. $R$ has finite fibers if and only if $R_{P}$ has finite fibers for each $P \in \operatorname{Spec}(R)$.

Proof. If $R$ does not have finite fibers, then for some overring $T$ of $R$ 
and some $P \in \operatorname{Spec}(R)$, we have that $\mathrm{fib}_{T}(P)$ is infinite. Passing to $R_{P} \subset T_{R \backslash P}$, we get that $\mathrm{fib}_{T_{R \backslash P}}\left(P R_{P}\right)$ is infinite.

The converse is immediate from Corollary 4.7.

Proposition 4.9. If $R$ has finite fibers, then $R$ is an inc-domain.

Proof. Deny. Then there exists an overring $T$ of $R$ with primes $Q_{1} \subsetneq Q_{2}$ such that $Q_{1} \cap R=Q_{2} \cap R(=P)$. By passing to $R_{P} \subset T_{R \backslash P}$, we may assume $R$ is local with maximal ideal $P$. Let $u \in Q_{2} \backslash Q_{1}$, and observe that $R \subset R[u]$ does not satisfy INC. The proof of Lemma 2.33 shows that fib ${ }_{R[u]}(P)$ is infinite, the required contradiction.

[7, Example 2.1] shows that the converse of Proposition 4.9 fails in general. We show next that, with the additional hypothesis that $R \subset \bar{R}$ has finite fibers, the converse becomes valid.

PROPOSITION 4.10. $R$ has finite fibers if and only if $R$ is an inc-domain and $R \subset \bar{R}$ has finite fibers.

Proof. The "only if" part is clear. Conversely, suppose that $R$ is an inc-domain which does not have finite fibers. Then there exist an overring $T$ of $R$ and a prime $P$ of $R$ such that fib ${ }_{T}(P)$ is infinite. Using the "rectangle argument", we see that fib $\overline{R T}_{T}(P)$ is infinite since $T \subset \bar{R} T$ satisfies LO. As $\bar{R} \subset \bar{R} T$ is an $i$-extension (since $\bar{R}$ is $\operatorname{Prüfer}_{\text {), }} \mathrm{fib}_{\bar{R}}(P)$ is infinite. Thus, $R \subset \bar{R}$ does not have finite fibers, completing the proof.

Proposition 4.11. $R$ is semilocal with finite fibers if and only if each overring of $R$ is semilocal.

Proof. Let $R$ be semilocal with finite fibers. By Proposition $4.9, \bar{R}$ is Prüfer; integrality shows that $\bar{R}$ is semilocal. If $T$ is an overring of $R$, one readily uses the fact that $\bar{R}$ is an $i$-domain (hence GD, hence treed) to show that $\bar{R} T$ is semilocal. By integrality, $T$ is then semilocal.

Conversely, assume that each overring of $R$ is semilocal. Suppose that $R$ does not have finite fibers; then there exist an overring $T$ of $R$ and a prime $P$ of $R$ such that $\mathrm{fib}_{T}(P)$ is infinite. We may assume that $R$ is local with maximal ideal $P$. By Lemma 2.33, each element of $\operatorname{fib}_{T}(P)$ is then a maximal ideal of $T$, contradicting the semilocality of $T$.

COROLlaRY 4.12. Let $n$ be a positive integer. If $R$ is a semilocal $i$-domain with exactly $n$ maximal ideals, then each overring of $R$ has at most $n$ maximal ideals.

Proof. Reexamine the first paragraph of the proof of Proposition 4.11. We now begin our main assault on the desired characterization. 
Lemma 4.13. $R$ is an integrally closed LH-domain if and only if $R$ is an open Prüfer domain.

Proof. Since LH-extensions are open, the "only if" half follows from Proposition 4.4. The converse is immediate, since any Prüfer domain is an U-domain.

LemmA 4.14. If $R$ is an LH-domain, then each overring of $R$ is an LH-domain.

Proof. Our strategy will be to show first that $\bar{R}$ is an LH-domain and then, using the "rectangle argument", to show that each overring of $R$ is an LH-domain.

Since $R$ is an LH-domain, Proposition 4.4 shows that $\bar{R}$ is a Prüfer domain. Now $R$ is open, hence semilocal, so that Corollary 4.2 gives that $\bar{R}$ is semilocal. To show that $\bar{R}$ is an LH-domain, it suffices by Lemma 4.13 and Theorem 3.16, to show that each branch of $\bar{R}$ is well-ordered under inclusion. Let $\left\{Q_{\alpha}: \alpha \in \Omega\right\}$ be a nonempty subset of a branch $[0, M]$ of $\bar{R}$. As $\left\{Q_{\alpha} \cap R: \alpha \in \Omega\right\}$ is a nonempty subset of some branch of $R$, we see that $\left\{Q_{\alpha} \cap R: \alpha \in \Omega\right\}$ has a first element, say $Q_{\beta} \cap R$, with $\beta \in \Omega$. Since $R$ is an inc-domain and $\bar{R}$ is treed, $Q_{\beta}$ is the first element of $\left\{Q_{\alpha}: \alpha \in \Omega\right\}$. Therefore $\bar{R}$ is an LH-domain.

Let $T$ be any overring of $R$. Then $T$ is an U-domain by Proposition 4.5, and $T$ is GD by Proposition 4.6. Using the same argument as above, we see that each branch of $T$ is well-ordered under inclusion. To complete the proof, it remains only to show that $T$ is semilocal. With the aid of the "rectangle argument", we first note that $\bar{R} T$ is semilocal by Corollary 4.12 , since $\bar{R}$ is a semilocal $i$-domain. Then, since $T \subset \bar{R} T$ is an integral extension, $T$ is semilocal, which completes the proof.

LEMMA 4.15. If $R$ is open with finite fibers and if each proper overring of $R$ is treed, then each overring of $R$ is open.

Proof. By Proposition 4.9, $R$ is an inc-domain. Let $T$ be an overring of $R$. By Proposition 4.11, $T$ is semilocal, by Proposition 2.32, $T$ is GD. One may argue as in Lemma 4.14 in order to verify that $T$ inherits from $R$ the property of having each of its branches well-ordered under inclusion. Hence $T$ is open.

THEOREM 4.16. The following are equivalent on $R$ :

(a) $R$ is an LH-domain.

(b) (i) $R$ has finite fibers.

(ii) $R$ is open.

(iii) Each proper overring of $R$ is treed.

(c) (i) $R$ is open. 
(ii) Each proper overring of $R$ is semilocal and has well-ordered branches under inclusion.

(d) Each overring of $R$ is open.

Proof. (a) $\Rightarrow$ (b) by Corollary 4.2 and Lemma 4.14 , while (a) $\Rightarrow$ (c) by Lemma 4.14. Lemma 4.15 gives (b) $\Rightarrow$ (d).

To prove (d) $\Rightarrow$ (a), assume (d). Observe that Propositions 4.11 and 4.9 imply that $R$ is an inc-domain. Since $R$ is open, it suffices to show that $R$ is an U-domain. Let $T$ be an arbitrary overring of $R$, and $Q \in \operatorname{Spec}(T)$. Let $U=$ $[0, Q]$, and consider the contraction $f: \operatorname{Spec}(T) \rightarrow \operatorname{Spec}(R)$. Then $U$ is open in $\operatorname{Spec}(T)$ since $T$ is open, and $f$ is injective on $U$ since $R \subset T$ satisfies INC; thus $f$ is a local homeomorphism. Hence, (d) $\Rightarrow(a)$.

To complete the proof, use Proposition 4.11 to deduce that (c) $\Rightarrow(b)$.

COROllary 4.17. $R$ is an LH-domain if and only if $R$ is semilocal and $R_{P}$ is an LH-domain for each $P \in \operatorname{Spec}(R)$.

Proof. In view of Lemma 4.14, the "only if" part is immediate. Conversely, assume that $R$ is semilocal and that $R_{P}$ is an LH-domain for each $P \in$ $\operatorname{Spec}(R)$. By Corollary $3.17, R$ is open, while Proposition 4.8 demonstrates that $R$ has finite fibers. By virtue of criterion (b) in Theorem 4.16, it is enough to show that each proper overring of $R$ is treed.

Let $T$ be a proper overring of $R$, and suppose that $T$ is not treed. Then there exists a maximal ideal $M$ of $T$ and primes $P$ and $Q$ of $T$ contained in $M$, such that $P$ and $Q$ are not comparable. Pass to $R_{M \cap R} \subset T_{M}$, and observe that $T_{M}$ is not treed. This contradicts the assumption that $R_{M \cap R}$ is an LH-domain, and completes the proof.

COROLlaRY 4.18. If $R$ is an LH-domain, then $R / P$ is an LH-domain for each $P \in \operatorname{Spec}(R)$.

Proof. Apply Theorem 4.16(d) combined with Corollary 3.19 and Proposition 2.1.

Remark 4.19. It follows immediately from Theorem 4.16 and Proposition 3.20 that a Noetherian domain $R$ is an LH-domain if and only if $R$ is semilocal and $\operatorname{dim}(R) \leqslant 1$.

Although we have just seen that Noetherian LH-domains are well understood, (4.20)-(4.22) will show that the situation for Noetherian LI-domains is more complicated.

Proposition 4.20. Let $u_{1}, \ldots, u_{n} \in(\mathrm{qf}(R) \backslash R)$. If $R$ is a Noetherian domain and $\operatorname{dim}(R)=1$, then $R \subset R\left[u_{1}, \ldots, u_{n}\right]$ is an LH-extension. 
Proof. Let $T=R\left[u_{1}, \ldots, u_{n}\right]$, and consider $f: X=\operatorname{Spec}(T) \rightarrow \operatorname{Spec}(R)$. Since $R$ is GD, it follows from [22, Theorem 8, p. 48] that $f$ is an open map, so that it now suffices to show that $f$ is a local injection. Let $I_{j}$ be the conductor of $u_{j}$ in $R$; set $I=\bigcap_{j=1}^{n} I_{j}$. Note that $0 \neq I \neq R$. As $R$ is 1-dimensional Noetherian, $I$ is contained in only finitely many primes of $R$, say $P_{1}, \ldots, P_{t}$. By slightly modifying the proof of [24, Lemma 2], one shows that, if $Q \in \operatorname{Spec}(R)$ and $I \not \subset Q$, then the cardinality of $\mathrm{fib}_{T}(Q)$ is one. To show that $f$ is a local injection, we consider two cases.

Case 1. Suppose $Q \in \mathrm{fib}_{T}\left(P_{i}\right)$ for some $\mathrm{i} \leqslant i \leqslant t$. By the Krull-Akizuki theorem [18, Theorem 93], $T$ is 1-dimensional Noetherian. Since $R \subset T$ has finite fibers [18, Exercise 21, p. 64], there exists $s \in T Q Q$ which belongs to every member of $\operatorname{fib}_{T}\left(P_{1}\right), \ldots$, fib $_{T}\left(P_{t}\right)$ except $Q$. Therefore, $Q$ is the only member of $\operatorname{fib}_{T}\left(P_{1}\right), \ldots, \mathrm{fib}_{T}\left(P_{t}\right)$ which is in $X_{s}$. Hence, $f$ is injective on $X_{s}$.

Case 2. Suppose $Q \in \operatorname{Spec}(T)$, such that $Q \notin \bigcup_{i=1}^{t} \mathrm{fib}_{T}\left(P_{i}\right)$. By the 1-dimensionality of $T$, we may choose $w \in T \backslash Q$ which belongs to every member of $\mathrm{fib}_{T}\left(P_{1}\right), \ldots, \mathrm{fib}_{T}\left(P_{t}\right)$. Then $f$ is injective on $X_{w}$, which completes the proof.

Proposition 4.21. Let $R$ be a semilocal Noetherian domain. Then $R$ is an LI-domain if and only if $\operatorname{dim}(R) \leqslant 1$.

Proof. If $R$ is an LI-domain, Proposition 4.4 shows that $R$ is an inc-domain; since $R$ is Noetherian, [11, Theorem 35.16] shows that $\operatorname{dim}(R) \leqslant 1$. The converse follows immediately from Remark 4.19.

We next present an example to show that an arbitrary 1-dimensional Noetherian domain need not be an LI-domain.

EXAMPLE 4.22. Let $R$ be the 1-dimensional local Noetherian domain in Example 2.17. Recall that $\bar{R}$ has exactly two maximal ideals, both of which necessarily lie over the unique maximal ideal of $R$. Let $F=\left\{R_{1}, R_{2}, R_{3}, \ldots\right\}$ where each $R_{i}=R$. Applying [17, Proposition 1] to $F$, we construct a 1-dimensional Noetherian domain $T$ with infinitely many primes, such that, for each nonzero $P \in \operatorname{Spec}(T)$, the cardinality of $\mathrm{fib}_{\bar{T}}(P)$ is greater than one. We claim that $T \subset \bar{T}$ fails to be an LI-extension. If not, let $0 \neq Q \in \operatorname{Spec}(\bar{T})$; then there exists an open set $U$ in $\operatorname{Spec}(\bar{T})$ such that $Q \in U$ and $\operatorname{Spec}(\bar{T}) \rightarrow \operatorname{Spec}(T)$ is injective on $U$. But, [2, Proposition 10, p. 98] combined with [22, Lemma 6.D, p. 41] implies that $\operatorname{Spec}(\bar{T}) \backslash U$ is a finite set. This contradicts the fact that the cardinality of each nonzero fiber is greater than one, to complete the proof.

REMARK 4.23. The preceding example shows that many of the basic properties which hold for the other classes of domains studied above, do not hold for the class of LI-domains. For example, Proposition 4.5 shows that LI-domains localize, while Proposition 4.21 and Example 4.22 combine to show that LI-domains need not globalize. Moreover, the LI analogue of Lemma 3.30 fails, as 
one sees by combining Proposition 4.20 with Example 4.22. Finally, using Example 4.22 and the Krull-Akizuki theorem, one has a domain $R$ such that $R \subset V$ is an LI-extension for each valuation overring $V$ of $R$, although $R$ is not an LI-domain. We have not as yet been able to determine whether valuation overring serve as test overrings for the LH property. (That overrings of the form $R\left[u_{1}, \ldots, u_{n}\right]$, with $u_{i} \in \mathrm{qf}(R)$, do not suffice, follows from (4.20) and (4.22).)

REMARK 4.24. Using Theorem 4.16 and Corollary 3.29 one deduces the following. Let $V$ be a valuation ring of the form $F+M$ and $R$ a subring of $F$. Then $R+M$ is an LH-domain if and only if $V$ is open and each ring $T$ satisfying $R \subset T \subset F$ is open.

We end this section by considering a special class of LH-extensions. For the sake of completeness, we first recall the following definition [21, p. 145]. Let $\underline{X}$ be a topological space which is pathwise connected and locally pathwise connected. A covering space of $\underline{X}$ is a pair consisting of a pathwise connected, locally pathwise connected space $\underline{\widetilde{X}}$ and a continuous map $p: \underline{\widetilde{X}} \rightarrow \underline{X}$ such that the following condition holds. Each point of $\underline{X}$ has a pathwise connected open neighborhood $U$ such that each path component of $p^{-1}(U)$ is mapped homeomorphically onto $U$ by $p$. [In particular, it is assumed that $p^{-1}(U)$ is nonempty.] It is readily proved that, if $(\underline{X}, p)$ is a covering space of $\underline{X}$, then $p$ is a local homeomorphism. Hence, it is natural to ask about covering spaces in our setting.

LemmA 4.25. If $R$ is a domain, then $\operatorname{Spec}(R)$ is contractible.

Proof. Define $H: \operatorname{Spec}(R) \times[0,1] \rightarrow \operatorname{Spec}(R)$ by $H(P, t)=P$ if $t=0$ and $H(P, t)=0$ otherwise. We claim that $H$ is continuous. Indeed, if $U$ is a nonempty open subset of $\operatorname{Spec}(R)$, then

$$
H^{-1}(U)=U \times[0,1] \cup \operatorname{Spec}(R) \times(0,1]
$$

which is open in $\operatorname{Spec}(R) \times[0,1]$. Thus, $H$ shows that $\operatorname{Spec}(R)$ is contractible to 0 .

REMARK 4.26. It is clear from Lemma 4.25 that, if $R$ is a domain, then $\operatorname{Spec}(R)$ is pathwise connected. Indeed, $\operatorname{Spec}(R)$ is then locally pathwise connected; for if $P$ and $Q$ are primes of $R$, one obtains a path from $P$ to $Q$, within any open set containing both $P$ and $Q$, via the continuous function $\phi:[0,1] \rightarrow$ $\operatorname{Spec}(R)$ given by $\phi(0)=P, \phi(1)=Q$ and $\phi(t)=(0)$ whenever $0<t<1$.

Proposition 4.27. Let $R$ and $T$ be domains, and let $p: \operatorname{Spec}(T) \rightarrow \operatorname{Spec}(R)$ be a continuous map. Then $(\operatorname{Spec}(T), p)$ is a covering space of $\operatorname{Spec}(R)$ if and only if $p$ is a homeomorphism.

Proof. The "if" half is trivial. For the converse, apply Lemma 4.25 and [21, Exercise 6.1, p. 160]. 
Before proving the final corollary of this section, we pause to make the following definition. An extension $R \subset T$ of domains is called a CS-extension (covering space extension) if $(\operatorname{Spec}(T), f)$ is a covering space of $\operatorname{Spec}(R)$, where $f$ is the contraction map. It is obvious from Observation 1 in Remark 3.40 that $R \subset T$ is a CS-extension for each overring $T$ of $R$ if and only if $R$ is a field.

COROLlARY 4.28. The following are equivalent on $R$ :

(a) $R$ is a local $i$-domain and $\operatorname{dim}(R) \leqslant 1$.

(b) $R \subset T$ is a CS-extension for each $u \in \mathrm{qf}(R)$ satisfying $T=R[u] \subsetneq \mathrm{qf}(R)$.

(c) $R \subset T$ is a CS-extension for each overring $T$ of $R$ satisfying $T \subsetneq \mathrm{qf}(R)$.

PRoof. (a) $\Rightarrow$ (c) is immediate, since (a) implies that any overring of $R$, other than $\mathrm{qf}(R)$, is 1-dimensional local. (c) $\Rightarrow$ (b) trivially, we shall show (b) $\Rightarrow$ (a) in order to complete the proof. Assume (b). In view of Proposition 4.27, we may apply Proposition 2.10 to show that $R$ is an $i$-domain. It now suffices to prove that $R$ has at most one nonzero prime. If $P, Q$ are distinct nonzero primes of $R$, one may, without loss of generality, select $r \in P \backslash Q$. Thus $R[1 / r]$ $\subsetneq \mathrm{qf}(R)$, since $Q$ survives in $R[1 / r]$. However, $P$ does not survive in $R[1 / r]$, contradicting the fact that $\operatorname{Spec}(R[1 / r]) \rightarrow \operatorname{Spec}(R)$ is surjective. This completes the proof.

REMARKS 4.29. (a) Example 2.17 illustrates that the (equivalent) conditions in Corollary 4.28 are not implied by the requirement that $R \subset V$ be a CS-extension for each valuation overring $V$ of $R$ other than $\mathrm{q}(R)$.

(b) Example 2.17 shows, i.a., that an LH-domain need not satisfy the equivalent conditions of Corollary 4.28 , although any domain satisfying those conditions is an LH-domain. Similarly, LH-domains form a proper subclass of the class of open domains, by virtue of Corollary 3.28.

5. Propen not open domains. Recall from $\S 3$, that a domain $R$ is called propen not open if $R$ is propen but not open. We shall presently find (in Example 5.11) many such domains. As in the previous sections, we shall develop basic machinery, which will then be applied to yield characterizations of propen not open domains in terms of previously studied classes of rings. For example, Corollaries 5.20 and 5.22 show how an arbitrary propen not open domain is built topologically by gluing the spectrum of an open domain to the spectrum of a local propen not open domain; moreover, Corollary 5.10 (together with Lemma 3.13) provides a ring theoretic characterization of local propen not open domains.

The terminology and notation of the previous sections will be used freely in this section, along with the following new definitions and notation. Let $R$ be a treed domain. Call the set $\{Q \in \operatorname{Spec}(R): Q \subset J(R)\}$ the trunk of $R$ and denote 
it by $\operatorname{tr}(R)$. Note that $\operatorname{tr}(R)$ is totally ordered, since $R$ is treed. Call the prime $\cup\{Q: Q \in \operatorname{tr}(R)\}$ the vertex of $R$ and let $v(R)$ denote that prime.

Unless otherwise mentioned, $R$ will always be a domain which is not a field.

PROPOSITION 5.1. $R$ is propen not open if and only if

(a) $R$ is $G D$.

(b) $[0, P]$ is open for each nonzero $P \in \operatorname{Spec}(R)$.

(c) No overring of $R$ other than $\mathrm{qf}(R)$ is a G-domain.

Proof. Assume that $R$ is propen not open. Then, (a) and (b) follow trivially. As for (c), let $T$ be an overring of $R$ such that $T \neq \mathrm{qf}(R)$. Since $R$ is not a $G$-domain, Lemma 3.8 shows that $T$ is not a $G$-domain.

Conversely, assume (a), (b) and (c). By the convention mentioned in the introduction to this section, $R \neq \mathrm{qf}(R)$; hence $R$ is not a $G$-domain, so that $R$ is not open. To complete the proof, we show that $R$ is propen. Let $T$ be an overring of $R$ with $T \neq \mathrm{qf}(R)$, and consider the image under the contraction $f: X=\operatorname{Spec}(T) \rightarrow \operatorname{Spec}(R)$ of $X_{t}$, where $t \in T$. We may assume $X_{t} \neq \varnothing$; moreover, $X_{t} \neq\{0\}$ since $T$ is not a $G$-domain. As in the proof of Proposition 3.2, conditions (a) and (b) imply that $f\left(X_{t}\right)=\bigcup_{\alpha \in \Omega}\left[0, P_{\alpha}\right]$, where each $P_{\alpha} \in$ $\operatorname{Spec}(R)$ and $\Omega$ is a nonempty indexing set. Without loss of generality, each $P_{\alpha} \neq 0$ since $X_{t} \neq\{0\}$; then condition (b) implies $f\left(X_{t}\right)$ is open, to complete the proof.

Conditions (a) and (b) of Proposition 5.1 have been encountered in earlier sections. In order to make Proposition 5.1 more applicable, we next focus attention on tools which will lead to a more thorough understanding of condition (c).

Lemma 5.2. Let $T$ be an overring of $R$ with $T \neq \mathrm{qf}(R)$, such that $R \subset T$ is an i-extension. If $R$ is propen not open, then $T$ is propen not open.

Proof. Argue as in Corollary 3.11.

LEMMA 5.3. If no valuation overring of $R$ other than $\mathrm{q}(R)$ is a G-domain, then no overring of $R$ other than $\mathrm{qf}(R)$ is a G-domain.

Proof. If not, let $T$ be a $G$-domain such that $R \subset T \subsetneq \mathrm{qf}(R)$. Select any valuation overring $V$ of $T$ other than $\mathrm{qf}(R)$. By [18, Theorem 20], $V$ is a $G$-domain, the desired contradiction.

LEMMA 5.4. If $R$ is propen not open, then $R$ has no nonzero primes of finite height.

Proof. It suffices to show that there are no primes of height one in $R$. This was done in the alternate proof of Corollary 3.5. 
Proposition 5.5. Let $R$ be GD. Then no overring of $R$ other than $\mathrm{qf}(R)$ is a G-domain if and only if $R$ has no nonzero primes of finite height.

Proof. Suppose that no overring of $R$ other than $\mathrm{qf}(R)$ is a $G$-domain. If $R$ has a nonzero prime $P$ of finite height then, since $R$ is treed, $R_{P}$ is also treed, whence $\operatorname{Spec}\left(R_{P}\right)$ is a finite set. Then [18, Exercise 3, p. 19] implies that $R_{P}$ is a $G$-domain, the desired contradiction.

For the converse, assume that $R$ has no nonzero primes of finite height. Let $\left\{M_{\alpha}\right\}_{\alpha \in \Omega}$ be the set of maximal ideals of $R$. If, for some $\alpha \in \Omega$, $\bigcap\left\{Q \in \operatorname{Spec}(R): Q \neq 0\right.$ and $\left.Q \subset M_{\alpha}\right\}$ were nonzero, it would be a height one prime, contrary to hypothesis. Therefore $R$ is a $G$-domain. To complete the proof, Lemma 5.3 shows that it suffices to establish that no valuation overring $V$ of $R$ other than $\mathrm{qf}(R)$ is a $G$-domain. Since $V$ is local treed, it is enough to show that $V$ has no nonzero primes of finite height.

Deny. Then $V$ has a prime $P$ of some positive finite height. Since $P \cap R$ is a nonzero prime of $R$, we conclude that $P \cap R$ does not have finite height. As $R \subset V$ satisfies GD, it follows that the height of $P$ is also infinite, a contradiction, completing the proof.

REMARK 5.6. If a nonzero prime $P$ of a domain $R$ has infinite height, $P$ need not contain an infinite chain of primes. However, if no nonzero prime of $R$ has finite height, then each nonzero prime of $R$ contains an infinite chain of primes.

Using the characterization in Proposition 5.1 along with Proposition 5.5, we give (in Corollary 5.10) a characterization for local propen not open domains.

Lemma 5.7. Let $R$ be local treed. Then $R$ is not a G-domain if and only if $R$ has no nonzero primes of finite height.

Proof. The "if" half is follows from the proof of Proposition 5.5. Conversely, assume $R$ is not a $G$-domain, but possesses a prime $P$ of positive finite height, $n$. Then there exists a chain of nonzero distinct primes $P_{1} \subsetneq \cdots \subsetneq P_{n-1}$ $\subsetneq P_{n}=P$. So $\bigcap\{Q \in \operatorname{Spec}(R): Q \neq 0\}=P_{1} \neq 0$, contradicting the fact that $R$ is not a $G$-domain.

Corollary 5.8. Let $R$ be local and GD. Then $R$ is not a G-domain if and only if no overring of $R$ other than $\mathrm{gf}(R)$ is a $G$-domain.

Proof. The "only if" half follows by combining Lemma 5.7 and Proposition 5.5. The converse is trivial.

EXAMPLE 5.9. We next construct a semilocal GD domain which is not a $G$-domain, and which possesses nonzero primes of finite height. (Hence, one cannot delete the assumption that $R$ is local in Lemma 5.7.) Let $X=\{(0,0)$, $(1 / 2,0),(1,0)\} \cup\{(0,1 / n): n \geqslant 1\}$. A partial order on $X$ may be given as 
follows: $(a, b) \leqslant(c, d)$ is and only if $a \leqslant c$ and $b \leqslant d$. By [20, Theorem 3.1], there exists a Bézout domain $R$ such that $\operatorname{Spec}(R)$ and $X$ are isomorphic as posets; that is, there is a bijection $f: \operatorname{Spec}(R) \rightarrow X$ such that both $f$ and $f^{-1}$ are order preserving. If $M$ is the maximal ideal of $R$ corresponding to $(0,1)$ in $X$, then

$$
\bigcap\{Q \in \operatorname{Spec}(R): Q \neq 0 \text { and } Q \subset M\}=0
$$

since $\lim _{n \rightarrow \infty}(1 / n)=0$. Thus $R$ is not a $G$-domain, However, $R$ does have (two) nonzero primes of finite height, corresponding to $(1 / 2,0)$ and $(1,0)$ in $X$.

Corollary 5.10. Let $R$ be local. Then $R$ is propen not open if and only if

(a) $R$ is $G D$.

(b) $[0, P]$ is open for each nonzero $P \in \operatorname{Spec}(R)$.

(c) $R$ is not a G-domain.

Proof. Combine Proposition 5.1 and Corollary 5.8.

Before proceeding further with the structure of propen not open domains, we give an example which helps motivate some of our later work.

EXAMPLE 5.11. For each positive integer $t$, we shall construct a propen not open domain $R$ with exactly $t$ maximal ideals. Let

$$
Y=\{(0,0)\} \cup\{(1 / n, 0): n \geqslant 1\} \cup\{(1+1 / n, 2-1 / n): 1 \leqslant n \leqslant t\} ;
$$

partially order $Y$ as in Example 5.9. Then [20, Theorem 3.1] provides a Bézout domain $R$ such that $\operatorname{Spec}(R)$ and $Y$ are isomorphic as posets. Note that $R$ has precisely $t$ maximal ideals, corresponding to $\{(1+1 / n, 2-1 / n): 1 \leqslant n \leqslant t\}$. By Proposition 5.5 , no overring of $R$ other than $\mathrm{qf}(R)$ is a $G$-domain. In order to prove that $R$ is propen not open, Proposition 5.1 now reduces us to showing that $[0, P]$ is open in $\operatorname{Spec}(R)$ for each nonzero $P \in \operatorname{Spec}(R)$. If $P$ is nonmaximal, we may appeal to Lemma 3.13. If $P$ is maximal, denote the remaining maximal ideals of $R$ by $M_{2}, \ldots, M_{t}$, and observe that $[0, P]^{\prime}=\left\{M_{2}, \ldots, M_{t}\right\}$ is closed in $\operatorname{Spec}(R)$, to complete the proof.

Before leaving this train of thought, we give a result which is motivated by Proposition 3.10 .

Proposition 5.12. $R$ is propen not open if and only if $R \subset V$ is open for each valuation overring $V$ of $R$ with $V \neq \mathrm{qf}(R)$ and $R \subset \mathrm{qf}(R)$ is not open.

Proof. We need only prove the "if" half. By [6, Theorem 1], $R$ is GD; by the proof of (b) $\Rightarrow$ (a) in Proposition 3.10, $R \subset R_{P}$ is open for each nonzero $P \in \operatorname{Spec}(R)$. By Proposition 5.1, we are reduced to showing that no overring of $R$ other than $\mathrm{qf}(R)$ is a $G$-domain. Note that $R$ is not a $G$-domain since 
$R \subset \mathrm{qf}(R)$ is not open. Thus, Lemma 3.8 shows that no valuation overring of $R$ other than $\mathrm{qf}(R)$ is a $G$-domain, and Lemma 5.3 applies to complete the proof.

Our next aim is to show how propen not open domains are built topolog. ically.

Proposition 5.13. If $R$ is propen not open, then there exists a nonzero prime contained in the Jacobson radical of $R$, that is, $\operatorname{tr}(R) \neq\{0\}$.

Proof. Since $R$ is propen not open, $R$ is semilocal and has no nonzero primes of finite height. We may assume that $R$ is not local; let $M_{1}, \ldots, M_{t}$ be the maximal ideals of $R$.

Suppose that, whenever $2 \leqslant i \leqslant t$, there exists a nonzero prime $P_{i}$ contained in $M_{1} \cap M_{i}$. If $P_{j}$ is the least of $P_{2}, \ldots, P_{t}$ (which are comparable since $R$ is treed), then $P_{j} \subset \bigcap_{k=1}^{t} M_{k}=J(R)$.

Hence, without loss of generality, we may suppose that 0 is the only prime of $R$ which is contained in $M_{1} \cap M_{2}$. As $R$ is propen not open, [0, $\left.M_{1}\right]$ is open in $\operatorname{Spec}(R)$, so that Lemma 3.13 supplies a unique prime $Q \subset M_{2}$ such that $Q$ is proper minimal over $\bigcup\left\{P: P \subset M_{1} \cap M_{2}\right\}$. Hence, $Q$ is a height one prime, a contradiction, which completes the proof.

Corollary 5.14. If $R$ is propen not open, then $\operatorname{tr}(R)$ is an infinite set.

Proof. Apply Proposition 5.13 and Remark 5.6.

REMARK 5.15. Unlike the situation for propen not open domains, the trunk of an open domain may consist merely of 0 . For example, if $X=\{(0,0)$, $(1 / 2,0),(1,0),(0,1 / 2),(0,1)\}$ is partially ordered as in Example 5.9, [20, Theorem 3.1] supplies a Bézout domain $R$ such that $\operatorname{Spec}(R)$ and $X$ are isomorphic posets. By Theorem 3.16, $R$ is open, although $\operatorname{tr}(R)=\{0\}$.

Lemma 5.16. Let $M_{1}, \ldots, M_{t}$ be the maximal ideals of a propen not open domain $R$. Then, for each $1 \leqslant i \leqslant t,\left[v(R), M_{i}\right]$ is well-ordered under inclusion.

Proof. Deny. Then ( $t>1$ and) for some $1 \leqslant j \leqslant t,\left[v(R), M_{j}\right]$ is not well-ordered under inclusion. Thus there exists a nonempty subset $\left\{Q_{\alpha}: \alpha \in \Omega\right\}$ $\subset\left[v(R), M_{j}\right]$ without a first element. Then $P=\bigcap\left\{Q_{\alpha}: \alpha \in \Omega\right\}$ is a prime of $R$ such that $v(R) \subset P \subsetneq M_{j}$. As $[0, P]$ is open, Lemma 3.13 supplies a unique prime $Q_{j} \subset M_{j}$ such that $Q_{j}$ is proper minimal over $\bigcup\left\{Q: Q \subset P \cap M_{j}\right\}=P$. In particular, $Q_{j} \in\left[v(R), M_{j}\right]$. Since $P \subsetneq Q_{j}$, there exists $\beta \in \Omega$ such that $Q_{j} \not \subset Q_{\beta}$, whence $Q_{\beta} \subsetneq Q_{j}$ by comparability. Minimality of $Q_{j}$ forces $P=Q_{\beta}$, which contradicts the assumption that $\left\{Q_{\alpha}: \alpha \in \Omega\right\}$ has no first element.

LEMMA 5.17. If $R$ is propen not open and $R / v(R)$ is $G D$, then $R / v(R)$ is open. 
Proof. $R / v(R)$ inherits from $R$ the property of being semilocal. Moreover, Lemma 5.16 implies that each branch of $R / v(R)$ is well-ordered under inclusion. Now apply Theorem 3.16 .

THEOREM 5.18. $R$ is propen not open and $R / v(R)$ is $G D$ if and only if $R$ is $G D, R_{v(R)}$ is propen not open, and $R / v(R)$ is open.

Proof. Assume that $R$ is propen not open. Then $R$ is clearly GD, $v(R)$ $\neq 0$ by Proposition 5.13, $R_{v(R)}$ is propen not open by Lemma 5.2 and $R / v(R)$ is open by Lemma 5.17 .

Conversely, assume $R$ is GD, $R_{v(R)}$ is propen not open, and $R / v(R)$ is open. Thus $R / v(R)$ is semilocal, so that $R$ is also semilocal; also, $v(R) \neq 0$ since $R_{v(R)}$ is propen not open. Since $R$ is GD, Propositions 5.1 and 5.5 reduce us to proving that $[0, P]$ is open for each nonzero $P \in \operatorname{Spec}(R)$ and that $R$ has no nonzero primes of finite height.

Suppose $P$ is a nonzero prime of $R$ with finite height. Since $P$ compares with $v(R)$ and since $R_{v(R)}$ is propen not open, Lemma 5.4 shows that $v(R) \subsetneq P$. As $R$ is treed, $v(R)$ is a nonzero prime with finite height, which contradicts Corollary 3.5 (as applied to $R_{v(R)}$ ). Therefore, $R$ has no nonzero primes of finite height.

We now consider three cases to prove that $[0, P]$ is open for each nonzero $P \in \operatorname{Spec}(R)$. Let $M_{1}, \ldots, M_{t}$ be the maximal ideals of $R$. We may assume $t>1$, so that $v(R)$ is not maximal.

Case 1. $0 \neq P \subsetneq v(R)$. Notice that $\left[0, P R_{v(R)}\right]$ is open in $\operatorname{Spec}\left(R_{v(R)}\right)$, since $R_{v(R)}$ is propen not open. Then Lemma 3.13 gives a unique prime $Q R_{v(R)} \subset v(R) R_{v(R)}$ such that $Q R_{v(R)}$ is proper minimal over $P R_{v(R)}$. Hence $Q \subset v(R)$ and $Q$ is proper minimal over $P$. Condition (a) of Lemma 3.13 shows that $[0, P]$ is open in $\operatorname{Spec}(R)$.

Case 2. $v(R) \subset P$ and $P$ not maximal. Since $R / v(R)$ is open, we have that $[v(R) / v(R), P / v(R)]$ is open in $\operatorname{Spec}(R / v(R))$; by Lemma 3.13, for each $1 \leqslant i \leqslant t$, there exists a unique prime $Q_{i} / v(R) \subset M_{i} / v(R)$ such that $Q_{i} / v(R)$ is proper minimal over $\bigcup\left\{Q / v(R): Q / v(R) \subset P / v(R) \cap M_{i} / v(R)\right\}$. For each $i, Q_{i} \subset M_{i}$; moreover, we claim that $Q_{i}$ is proper minimal over $\bigcup\left\{Q^{\prime}: Q^{\prime} \subset P \cap M_{i}\right\}$. (Note that $Q_{i}$ will then be the unique such, as $R$ is treed.)

First, we show that $\bigcup\left\{Q^{\prime}: Q^{\prime} \subset P \cap M_{i}\right\} \subsetneq Q_{i}$. If not, comparability gives $Q_{i} \subset \bigcup\left\{Q^{\prime}: Q^{\prime} \subset P \cap M_{i}\right\}$, so that $Q_{i} / v(R) \subset P / v(R) \cap M_{i} / v(R)$, contradicting the fact that $Q_{i} / v(R)$ is proper minimal over $\bigcup\{Q / v(R): Q / v(R) \subset P / v(R) \cap$ $\left.M_{i} / v(R)\right\}$. Hence $\bigcup\left\{Q^{\prime}: Q^{\prime} \subset P \cap M_{i}\right\} \subsetneq Q_{i}$.

As for minimality, suppose that $\bigcup\left\{Q^{\prime}: Q^{\prime} \subset P \cap M_{i}\right\} \subsetneq Q_{i}^{\prime} \subset Q_{i}$. Observe that $v(R) \subset \bigcup\left\{Q^{\prime}: Q^{\prime} \subset P \cap M_{i}\right\}$, since $v(R) \subset P \cap M_{i}$. We claim that $\bigcup\left\{Q / v(R): Q / v(R) \subset P / v(R) \cap M_{i} / v(R)\right\} \subsetneq Q_{i}^{\prime} / v(R) \subset Q_{i} / v(R)$. If not, then 
$Q_{i}^{\prime} / v(R) \subset \bigcup\left\{Q / v(R): Q / v(R) \subset P / v(R) \cap M_{i} / v(R)\right\}$, since $R / v(R)$ is treed; then $Q_{i}^{\prime} \subset P$, a contradiction. Thus, by the minimality of $Q_{i} / v(R)$, we have $Q_{i}^{\prime} / v(R)=$ $Q_{i} / v(R)$, so that $Q_{i}^{\prime}=Q_{i}$. By condition (a) of Lemma 3.13, [0,P] is open.

Case 3. $P$ maximal. Argue as in Case 2 , with the aid of condition (b) of Lemma 3.13.

This completes the proof.

Using the above theorem, we shall now show (in Corollary 5.20 and Corollary 5.22) that the spectrum of a propen not open domain may be constructed by gluing the spectrum of an open domain to the top of the spectrum of a local propen not open domain.

LEMma 5.19. If $R$ is treed, then $\operatorname{Spec}(R)$ is homeomorphic to $\operatorname{Spec}\left(R_{v(R)}\right)$ $\sqcup \operatorname{Spec}(R / v(R)) /\left(v(R) R_{v(R)}=v(R) / v(R)\right)$.

Proof. Apply [15, Lemma 3.13].

CoROLLARY 5.20. If $R$ is propen not open and $R / v(R)$ is $G D$, then $\operatorname{Spec}(R)$ is homeomorphic to

$$
\left(\operatorname{Spec}\left(R_{v(R)}\right) \sqcup \operatorname{Spec}(R / v(R))\right) /\left(v(R) R_{v(R)}=v(R) / v(R)\right),
$$

where $R_{v(R)}$ is local propen not open and $R / v(R)$ is open.

Proof. Combine Theorem 5.18 and Lemma 5.19.

Corollary 5.22 will present a decomposition of $\operatorname{Spec}(R)$, similar to the preceding, for an arbitrary propen not open $R$. First, a lemma:

LEMMA 5.21. Let $R$ and $S$ be semilocal treed domains. If $f: \operatorname{Spec}(R) \rightarrow$ $\operatorname{Spec}(S)$ is an isomorphism as posets, then $f$ is a homeomorphism.

Proof. Let $M_{1}, \ldots, M_{t}$ be the maximal ideals of $R$, and let $Y=\operatorname{Spec}(S)$. We first show that $f$ is continuous. Let $0 \neq s \in S$; without loss of generality, $Y_{s} \neq Y$. Moreover, $\left(Y_{s}\right)^{\prime}=V(J)$ for some ideal $J$ of $S$. Relabel so that $J \subset$ $\bigcup_{i=1}^{k} f\left(M_{i}\right)$ and $J \not \subset \bigcup_{k<j<t} f\left(M_{j}\right)$. If $Q_{i}=\bigcap\left\{Q \in Y: J \subset Q \subset f\left(M_{i}\right)\right\}$, one verifies that $V(J)=\bigcup_{i=1}^{k} V\left(Q_{i}\right)$, so that $\left(f^{-1}\left(Y_{s}\right)\right)^{\prime}=\bigcup_{i=1}^{k} V\left(f^{-1}\left(Q_{i}\right)\right)$.

Similarly, $f^{-1}$ is continuous and the proof is complete.

COROLlaRY 5.22. Let $R$ be propen not open. Then there exists a propen not open Bézout domain $S$ and an inclusion-preserving homeomorphism of $\operatorname{Spec}(R)$ onto $\operatorname{Spec}(S)$. Any such homeomorphism induces homeomorphisms of $\operatorname{Spec}\left(R_{v(R)}\right)$ with $\operatorname{Spec}\left(S_{v(S)}\right)$ and of $\operatorname{Spec}(R / v(R))$ with. $\operatorname{Spec}(\operatorname{S} / v(S))$. Hence $\operatorname{Spec}(R)$ is homeomorphic to a quotient space of the disjoint union of the spectra of a local propen not open domain and of an open domain.

Proof. By [20, Theorem 3.1], there exist a Bézout domain $S$ and a poset isomorphism $f: \operatorname{Spec}(R) \rightarrow \operatorname{Spec}(S)$. Since $S$ is GD and has no nonzero primes 
of finite height, the task of showing that $S$ is propen not open is reduced, by Propositions 5.1 and 5.5, to showing that $[0, P]$ is open in $\operatorname{Spec}(S)$ for each nonzero $P \in \operatorname{Spec}(S)$.

If $P$ is a nonzero prime of $S$, then $f^{-1}(P) \neq 0$, so that $\left[0, f^{-1}(P)\right]$ is open in $\operatorname{Spec}(R)$. However, $f$ is a homeomorphism by Lemma 5.21 , so that $[0, P]=$ $f\left(\left[0, f^{-1}(P)\right]\right)$ is indeed open in $\operatorname{Spec}(S)$. Therefore, $S$ is propen not open. As $S$ is Bézout, Proposition 2.1 implies that $S / v(S)$ is GD; moreover, $S_{v(S)}$ and $S / v(S)$ are propen not open and open respectively, by Theorem 5.18 .

As mentioned in Lemma 5.19, we may identify (up to inclusion-preserving homeomorphisms) $\operatorname{Spec}\left(R_{v(R)}\right)$ with $\operatorname{tr}(R)$ and $\operatorname{Spec}(R / v(R))$ with $V(v(R))$. Similar comments hold for $\operatorname{Spec}\left(S_{v(S)}\right)$ and $\operatorname{Spec}(S / v(S))$. Since $f(v(R))=v(S)$, the assertions about induced homeomorphisms are easily verified. The final conclusion follows by applying Corollary 5.20 to $S$.

EXAMPLE 5.23. The following example shows that one cannot delete the "semilocal" hypothesis in Lemma 5.21. In [12, Example 1, p. 279], a domain $R$ is constructed with the following properties: $\operatorname{dim}(R)=1$, the cardinality of $\operatorname{Spec}(R)$ is $c$ (i.e., that of the real numbers), and $R$ is a $G$-domain. Let $x$ be an indeterminate over $C$, the complex numbers. By Hilbert's Nullstellensatz, there is a poset isomorphism $f$ of $\operatorname{Spec}(R)$ onto $\operatorname{Spec}(C[x])$. Now, [18, Theorem 21] shows that $C[x]$ is not a $G$-domain. Thus $f$ is not a homeomorphism, since $\{0\}$ is open in $\operatorname{Spec}(R)$ and $f(\{0\})=\{0\}$ is not open in $\operatorname{Spec}(C[x])$.

The next example shows that an additional hypothesis is needed to obtain the propen not open analogue of Corollary 3.17. The appropriate analogue is given in Proposition 5.25.

EXAMPLE 5.24. Let $X=\{(0,0)\} \cup\{(0,1 / n): n \geqslant 1\} \cup\{(1 / n, 0): n \geqslant 1\}$, partially ordered as in Example 5.9. Again by [20, Theorem 3.1], there is a Bézout domain $R$ such that $\operatorname{Spec}(R)$ and $X$ are isomorphic as posets. $A s \operatorname{tr}(R)=$ $\{0\}, R$ fails to be propen not open. Moreover, $R$ is not a $G$-domain; therefore $R$ is not propen. We claim that $R_{P}$ is propen not open for each nonzero prime $P$ of $R$. Indeed, $R_{P}$ is a valuation domain since $R$ is Bézout, and the claim follows readily from Corollary 5.10 and Lemma 3.13 .

PROPOSITION 5.25. The following are equivalent on $R$ :

(a) $R$ is propen not open.

(b) $R_{P}$ is propen not open for each nonzero $P \in \operatorname{Spec}(R), R$ is semilocal and $v(R) \neq 0$.

(c) $R_{M}$ is propen not open for each maximal ideal $M$ of $R, R$ is semilocal and $v(R) \neq 0$.

Proof. Lemma 5.2, Corollary 3.4 and Proposition 5.13 unite to show that (a) $\Rightarrow(b)$, while (b) $\Rightarrow$ (c) trivially. To show (c) $\Rightarrow(a)$, assume (c). It is 
clear (cf. [5, Lemma 2.1]) that $R$ is GD. As in the proof of Theorem 5.18, it is enough to show that $[0, P]$ is open in $\operatorname{Spec}(R)$ for each nonzero $P \in \operatorname{Spec}(R)$, and that no nonzero prime of $R$ has finite height.

Let $M_{1}, \ldots, M_{t}$ be the maximal ideals of $R$. Let $P$ be a nonzero (possibly maximal) prime of $R$. We shall after considering two possibilities, show that $[0, P]$ is open in $\operatorname{Spec}(R)$, by an appeal to Lemma 3.13.

Case 1. $P \subsetneq M_{j}$. Then $\bigcup\left\{Q: Q \subset P \cap M_{j}\right\}=P$. As $R_{M_{j}}$ is propen not open, [0,PR $M_{j}$ ] is open in $\operatorname{Spec}\left(R_{M_{j}}\right)$, and so Lemma 3.13 yields a unique prime $Q_{j} R_{M_{j}} \subset M_{j} R_{M_{j}}$ such that $Q_{j} R_{M_{j}}$ is proper minimal over $\bigcup\left\{N R_{M_{j}}: N R_{M_{j}} \subset\right.$ $\left.P R_{M_{j}} \cap M_{j} R_{M_{j}}\right\}=P R_{M_{j}}$. Hence, $Q_{j}$ is the unique prime of $R$ which is contained in $M_{j}$ and which is proper minimal over $P$.

Case 2. $P \not \subset M_{j}$. Then $v(R) \subsetneq P$. Now $N_{j}=\bigcup\left\{Q: Q \subset P \cap M_{j}\right\}$ is a prime ideal containing $v(R)$. Moreover, $N_{j} \subsetneq M_{j}$; otherwise, $N_{j}=M_{j}$, so that $M_{j}=P$, a contradiction. Hence $v(R) R_{M_{j}} \subset N_{j} R_{M_{j}} \subsetneq M_{j} R_{M_{j}}$. As $v(R) \neq 0$, we have $\left[0, N_{j} R_{M_{j}}\right] \neq\{0\}$, and so the propenness of $R_{M_{j}}$ implies that $\left[0, N_{j} R_{M_{j}}\right]$ is open. By Lemma 3.13, there exists a unique prime $Q_{j} R_{M_{j}}$ of $R_{M_{j}}$ which is proper minimal over $\bigcup\left\{N R_{M_{j}}: N R_{M_{j}} \subset N_{j} R_{M_{j}} \cap M_{j} R_{M_{j}}\right\}=N_{j} R_{M_{j} \cdot}$. Thus $Q_{j}$ is the unique prime of $R$ which is contained in $M_{j}$ and is proper minimal over $N_{j}$.

By Lemma 3.13, the preceding two cases combine to prove that $[0, P]$ is open in $\operatorname{Spec}(R)$ for each nonzero $P \in \operatorname{Spec}(R)$.

To complete the proof, we show that $R$ has no nonzero primes of finite height. If, on the contrary, $R$ possesses a nonzero prime $P$ of finite height, then $P \subset M_{j}$ for some $1 \leqslant j \leqslant t$, so that $P R_{M_{j}}$ is a nonzero prime of finite height in $R_{M_{j}}$. In view of Propositions 5.1 and 5.5, this is a contradiction, which completes the proof.

As with the classes of rings studied in the earlier sections, propen not open domains may be obtained by the " $D+M$ construction". We close with some results along these lines. As in the previous sections, the proofs are deleted.

Proposition 5.26. Let $V$ be a valuation ring (not a field) of the form $F+M$, where $F$ is a field and $M$ is the maximal ideal of $V$. Let $S$ be a subring of $F$. Then $S+M$ is propen not open if and only if $S$ is open and $V$ is propen not open.

COROllaRy 5.27. Let $V$ be a valuation ring (not a field) of the form $F+M$, and let $S$ be a subring of $F$. Then every overring of $S+M$ other than $\mathrm{qf}(S+M)$ is propen not open if and only if $V$ is propen not open and every ring $T$ satisfying $S \subset T \subset F$ is open.

\section{BIBLIOGRAPHY}

1. E. Bastida and R. Gilmer, Overrings and divisorial ideals of rings of the form $D+$ M, Michigan Math. J. 20 (1973), 79-95. MR 48 \#2138.

2. N. Bourbaki, Commutative algebra, Addison-Wesley, Reading, Mass., 1972. 
3. J. Dawson and D. E. Dobbs, On going down in polynomial rings, Canad. J. Math. 26 (1974), 177-184. MR 48 \#8490.

4. D. E. Dobbs, On going down for simple overrings, Proc. Amer. Math. Soc. 39 (1973), 515-519. $439-458$.

5. - On going down for simple overrings. II, Comm. Algebra 1 (1974),

6. D. E. Dobbs and I. J. Papick, On going down for simple overrings. III, Proc. Amer. Math. Soc. 54 (1976), 35-38. (submitted).

7. D. E. Dobbs, Ascent and descent of going-down rings for integral extensions

8. J. Dugundji, Topology, Allyn and Bacon, Boston, Mass., 1966. MR 33 \#1824.

9. D. Ferrand, Morphismes entiers universellement ouverts (manuscript).

10. R. W. Gilmer, Jr. and W. J. Heinzer, Intersections of quotient rings of an integral domain, J. Math. Kyoto Univ. 7 (1967), 133-150. MR 36 \#6397.

11. R. W. Gilmer, Jr., Multiplicative ideal theory, Queen's Papers in Pure and Appl.

Math., no. 12, Queen's University, Kingston, Ont., 1968. MR 37 \#5198.

12. The pseudo-radical of a commutative ring, Pacific J. Math. 19 (1966),

275-284. MR 34 \#4294.

13. - Prïfer-like conditions on the set of overrings of an integral domain,

Conf. on Commutative Algebra (Univ. Kansas, Lawrence, Kan., 1972), Lecture Notes in

Math., vol. 311, Springer-Verlag, Berlin, 1973, pp. 90-102. MR 49 \#5000.

14. R. W. Gilmer, Jr. and J. Huckaba, $\Delta$-rings, J. Algebra 28 (1974), 414-432.

15. B. Greenberg, Ph.D. Dissertation, Rutgers University, New Brunswick, N. J., 1973.

16. A. Grothendieck and J. Dieudonné, téments de géométrie algébrique. IV, Inst.

Hautes Etudes Sci. Publ. Math. No. 20 (1964). MR 30 \#3885.

17. M. Hochster, Non-openness of loci in Noetherian rings, Duke Math. J. 40 (1973), 215-219. MR 47 \#215.

18. I. Kaplansky, Commutative rings, Allyn and Bacon, Boston, Mass., 1970. MR 40 \#7234.

19. Topics in commutative ring theory, University of Chicago, Chicago, III. (mimeographed notes).

20. W. J. Lewis, The spectrum of a ring as a partially ordered set, J. Algebra 25 (1973), 419-434. MR 47 \#3361.

21. W. S. Massey, Algebraic topology: An introduction, Harcourt, Brace and World, New York, 1967. MR 35 \#2271. \#1813.

22. H. Matsumura, Commutative algebra, Benjamin, New York, 1970. MR 42

23. S. McAdam, Going down in polynomial rings, Canad. J. Math. 23 (1971), 704711. MR 43 \#6202.

24. - Going down, Duke Math. J. 39 (1972), 633-636. MR 47 \#220.

25. - Going down and open extensions, Canad. J. Math. (to appear).

26. - Simple going down, J. London Math. Soc. (to appear).

27. M. Nagata, Local rings, Interscience Tracts in Pure and Appl. Math., no. 13, Interscience, New York, 1962. MR 27 \#5790.

28. I. J. Papick, Classes of going-down domains, Ph. D. Dissertation, Rutgers University, New Brunswick, N. J., 1974.

29. M. Raynaud, Anneaux locaux henséliens, Lecture Notes in Math., vol. 169, Springer-Verlag, Berlin and New York, 1970. MR 43 \#3252.

30. F. Richman, Generalized quotient rings, Proc. Amer. Math. Soc. 16 (1965), 794-799. MR 31 \#5880.

DEPARTMENT OF MATHEMATICS, RUTGERS UNIVERSITY, HILL CENTERBUSCH CAMPUS, NEW BRUNSWICK, NEW JERSEY 08903 York 11530

Current address: Department of Mathematics, Adelphi University, Garden City, New 